\title{
Pressure effect on the mechanical, electronic and thermodynamic properties of $\mathrm{Ba}_{2} \mathrm{Bi}_{3}$ : first-principle calculations
}

\author{
Jin-Jin Cao*, Xiao-Fan Gou \\ College of Mechanics and Materials, Hohai University, Nanjing, Jiangsu 210098, China
}

\begin{abstract}
The newly discovered $\mathrm{Ba}_{2} \mathrm{Bi}_{3}$ phase has attracted attentions in search of new superconductors, mainly because of the layered structure with two-dimensional superconducting Bi planes intercalated by $\mathrm{Ba}$ atoms. To clearly reveal what features the layered structure of the $\mathrm{Ba}_{2} \mathrm{Bi}_{3}$ phase correlates with, especially under pressure, we have systematically investigated the structural, mechanical, electronic and thermodynamic properties under the equivalent hydrostatic pressures up to $14 \mathrm{GPa}$ by means of first-principle calculations with the Local Density Approximation (LDA) and Generalized Gradient Approximation (GGA) approaches. The results indicate that the pressure effect on the crystal structure is performed as a compression of the unit-cell volume, mainly along the $c$-axis. Specifically for the mechanical related properties, the elastic constants and polycrystalline elastic moduli monotonically increase with the pressure. The calculated ductile factors show that the $\mathrm{Ba}_{2} \mathrm{Bi}_{3}$ lies in between the brittle and ductile border line at $0 \mathrm{GPa}$ and the pressure makes the ductility enhancement. Besides, the anisotropic properties of the $\mathrm{Ba}_{2} \mathrm{Bi}_{3}$ under various pressures are discussed. The electronic energy band structure and density of states under pressure are analyzed. Moreover, the pressure and temperature dependence of the thermodynamic properties through the quasi-harmonic Debye model, together with the pressure dependence of the Debye temperature calculated from elastic constants has been obtained. The calculated results of the variation of the Debye temperature and total density of states at Fermi level show that the pressure can lead to the superconducting transition temperature $\left(T_{c}\right)$ of the $\mathrm{Ba}_{2} \mathrm{Bi}_{3}$ decreasing.
\end{abstract}

Keywords: $\mathrm{Ba}_{2} \mathrm{Bi}_{3}$ superconductor; Mechanical properties; Electronic properties; Thermodynamic properties; First-principle calculations.

\footnotetext{
* Corresponding author. E-mail address: cj2lang@126.com .
} 


\section{Introduction}

Continuously exploring new superconductors keeps always the research interest in solid-state physics. Recently, the discovery of the bismuth binary compound superconductors has excited a greater enthusiasm [1-5] for their potential of uncovering the unconventional superconductivity and indicating a possible way to induce new superconductors with higher superconducting transition temperature. The $\mathrm{Ba}_{2} \mathrm{Bi}_{3}$ phase, as one of the bismuth binary compounds, was revealed of its superconductivity with a transition temperature $\left(T_{c}\right)$ of $4.4 \mathrm{~K}$ recently $[5,6]$, even had been already discovered since 2004 [7]. Different from other bismuth binary superconductors, the $\mathrm{Ba}_{2} \mathrm{Bi}_{3}$ has an attractive character of the layered structure with two-dimensional superconducting $\mathrm{Bi}$ planes intercalated by $\mathrm{Ba}$ atoms. The layered materials, typically such as the high-temperature cuprates and iron-based superconductors, have been a new fertile ground for the discovery of new superconductors. It has been found that the pressure can induce some novel superconductivities $[8,9]$ and also has some significant effects on other physics of layered structures. The layered $\mathrm{Ba}_{2} \mathrm{Bi}_{3}$, as a parent phase, should show new physics under compression [6]. Besides, the layered $\mathrm{Ba}_{2} \mathrm{Bi}_{3}$ is extracted from the heavier end of the periodic table of elements and can be regarded as another analogue of cuprates. Therefore, it can play an important role in the comparison study when exploring the physical properties of the conventional and new high-temperature cuprates, especially under pressure. Thus, it's very significant to deeply understand the correlation of the underlying physics of the $\mathrm{Ba}_{2} \mathrm{Bi}_{3}$ with its unique structure under high pressures, not only for seeking new superconductors but also for related potential applications.

To the best of our knowledge, so far there have been only a few investigations on the $\mathrm{Ba}_{2} \mathrm{Bi}_{3}$. The structural parameters and electron density of states were firstly obtained by Siméon Ponou and Thomas F. Fässler [7]. Then the superconductivity under pressures up to $1.5 \mathrm{GPa}$ was explored by A Iyo et al [5], and with doping $\mathrm{Sb}$ into the Bi sites was also investigated by Takeshi Yajima et al [10]. However, few studies of the mechanical, electronic and thermodynamic properties under higher 
pressures have ever been experimentally or theoretically carried out.

Generally, the elastic constants can quantify the response of materials to an applied macroscopic stress and offer the information regarding the structural stability, strength, ductility and anisotropy as well as Debye temperature. The energy band structure and density of states help to reveal the underlying mechanism of the physics of materials at microscale. Especially, the details of density of states at Fermi level are conducive to understanding the mechanism of superconductivity. The thermodynamic parameters, including the heat capacity (providing essential information about the vibrational properties), thermal expansion coefficient (reflecting the shape response to temperature), and Grüneisen parameter (describing the anharmonic effects of crystal lattice thermal vibration) can extend the knowledge of materials' performance under extremely conditions. Therefore, to know more about the mechanical, electronic and thermodynamic properties of the layered $\mathrm{Ba}_{2} \mathrm{Bi}_{3}$ will be highly desirable.

To obtain a deep insight of the $\mathrm{Ba}_{2} \mathrm{Bi}_{3}$ superconductor, in this work, we focus on the equivalent hydrostatic pressure effect on the structural, mechanical, electronic and thermodynamic properties from first-principle calculations. Finally, on the calculated electron density of states at Fermi level and Debye temperature, the pressure effect on the superconducting transition temperature of the $\mathrm{Ba}_{2} \mathrm{Bi}_{3}$ is further predicted.

\section{Computational methods}

The crystalline structure of the $\mathrm{Ba}_{2} \mathrm{Bi}_{3}$ is an orthorhombic structure with space group of Immm (No. 71). Each unit cell of the $\mathrm{Ba}_{2} \mathrm{Bi}_{3}$ consists of four $\mathrm{Ba}$ atoms and six $\mathrm{Bi}$ atoms. $\mathrm{Ba}$ atoms occupy the $4 j$ site $\left(0.5,0, \mathrm{Ba}_{z}\right), \mathrm{Bi}$ atoms occupy the $2 a$ site ( 0 , $0,0)$ and $4 h$ site $\left(0.5, \mathrm{Bi}(2)_{y}, 0\right)[5,7,10]$. The orthorhombic $\mathrm{Ba}_{2} \mathrm{Bi}_{3}$ exhibits layered structure with Two-dimensional superconducting Bi planes intercalated by $\mathrm{Ba}$ atoms, as shown in Fig. 1. The experimental lattice parameters under ambient pressure and room temperature are determined as $a=4.813 \AA, b=7.994 \AA$ and $c=9.971 \AA$ [5]. The calculations of the structural, mechanical and thermodynamic properties are performed by employing the plane wave pseudopotential (PW-PP) method within the framework of density functional theory which is realized in the computing package 
Quantum-Espresso [11] (QE). The electronic exchange-correlation energy is treated in the scheme of the Local Density Approximation (LDA) [12, 13] and early Perdew-Bruke-Ernzerhof (PBE) [14] as well as newly PBESOL [15] versions of Generalized Gradient Approximation. The electron-ion interaction is described by using scalar relativistic ultra-soft pseudopotentials with non-linear core correction contained in the GBRV library [16] which is efficient and accurate to produce lattice constants, bulk modulus and energy [17]. Based on convergence tests, the calculations are made with wave-function and charge-density cutoffs of $40 \mathrm{Ry}$ and $400 \mathrm{Ry}$, respectively. The Brillouin zone (BZ) is sampled by using the Monkhorst-Pack scheme [18] with $16 \times 10 \times 8 k$-mesh for the calculations of the structure optimization and self-consistent energy. For ionic minimization, the convergence threshold on total energy is $1.0 \mathrm{e}-5$ Ry and that on forces is $1.0 \mathrm{e}-4 \mathrm{Ry} / \mathrm{Bohr}$.

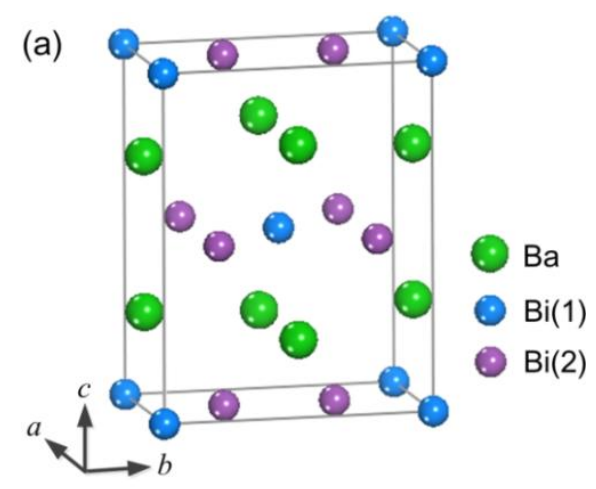

(b)

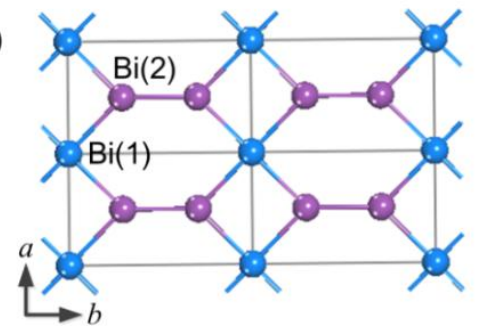

Fig. 1. (a) Crystal structure of the $\mathrm{Ba}_{2} \mathrm{Bi}_{3}$, where green, blue and purple spheres represent $\mathrm{Ba}$, $\mathrm{Bi}(1)$ and $\mathrm{Bi}(2)$, respectively. (b) A schematic illustration of the Bi ribbon nets viewed along the $c$-axis. Here, $a, b$, and $c$ are in Cartesian coordinate system.

As for electronic properties, the ELK code [19], an all-electron full-potential linearised augmented-plane wave (FP-LAPW) method, is used to determine the ground-state electronic structure. The lattice constants and internal atom coordinates 
are derived from the results on the PBE calculations in the scheme of Quantum-Espresso. The electronic calculations are made with a cutoff for plane-wave (in the interstitial region) determined by $|G+K|_{\max }=7.0 / R_{\mathrm{mt}}$, where the $R_{\mathrm{mt}}$ is the average muffin-tin radius. Muffin-tin radii of $2.8 \mathrm{Bohr}$ is used both for $\mathrm{Ba}$ and $\mathrm{Bi}$. Convergence is obtained on a $19 \times 12 \times 9$ mesh grid giving $350 k$-points for unit cell and $20 \times 20 \times 20$ giving $1160 k$-points for primitive cell in the irreducible BZ. The PBE [14] approximation is used for exchange-correlation effects among the electrons.

We remark that there are no more available experimental or theoretical results of the orthorhombic $\mathrm{Ba}_{2} \mathrm{Bi}_{3}$, here we use LDA, PBE and PBESOL approaches to ensure more reliable conclusions in the calculations of structural, mechanical and thermodynamic properties. The QE code is regarded as a high efficient tool to investigate structural, mechanical and thermodynamic properties which are derived from the total energies of structures. As the pseudopotential in GBRV library has not been tested for the electronic properties [16], another code is imperative for the electronic related researches. Specially, it's usually considered that the all-electron calculations can produce more accurate electronic structures, so the ELK code is employed. In addition, due to the fact that the structural information of the $\mathrm{Ba}_{2} \mathrm{Bi}_{3}$ is experimentally incomplete and that the structure optimization through employing all-electron calculations are expensive, the optimized structural parameters obtained from PBE calculations in the scheme of QE code under various pressures are used for the investigation of pressure effect on the electronic properties. It has been tested that the QE code with GBRV library and ELK code can both produce similar and reliable results of energy and bulk modulus [17], which means that the initial structures under high pressures obtained in this way for electronic calculations are reliable. Hopefully, the variation of related arguments can give reasonable results of the electronic properties with the pressure. Moreover, because the PBE calculations give closer related results to the experimental data (discussed below), so only the PBE calculations are performed in the electronic related studies. The combination of PW-PP and FP-LAPW methods elevates the efficiency and ensures more accurate results of electronic properties. 


\section{Results and discussion}

\subsection{Crystal structure}

In this work, the lattice parameters and internal atom coordinates are fully optimized without any restrictions under various pressures. The equilibrium lattice parameters and atomic coordinates of the $\mathrm{Ba}_{2} \mathrm{Bi}_{3}$ at $0 \mathrm{GPa}$ and $0 \mathrm{~K}$ are listed in Table 1, together with available experimental data $[5,7,10]$. It can be seen that the lattice constants under $0 \mathrm{GPa}$ appear to be in a reasonable accordance with available experimental data. The absolute error relative to experimental data is less than $3.4 \%$ on the LDA and less than $2.6 \%$ on the PBE and PBESOL, which are acceptable for employing the density functional theory calculations. Though the accuracy between PBE and PBESOL is comparable, the absolute error of the density and Debye temperature calculated on PBE is smaller at $0 \mathrm{GPa}$ (discussed below). Therefore the PBE can give more accurate results than PBESOL for the related researches of the $\mathrm{Ba}_{2} \mathrm{Bi}_{3}$. Due to the fact that there's no more available data for comparison, the pressure effect on the related properties is calculated on the PBE approach.

For internal atom coordinates, as the $\mathrm{Ba}_{2} \mathrm{Bi}_{3}$ is the parent phase of the $\mathrm{Ba}_{2} \mathrm{BiSb}_{2}$ and both compounds have similar layered structures, the value of $\mathrm{Ba}_{z}$ of the $\mathrm{Ba}_{2} \mathrm{Bi}_{3}$ should be close to that of $\mathrm{Ba}_{2} \mathrm{BiSb}_{2}$. The $\mathrm{Ba}_{z}$ of $\mathrm{Ba}_{2} \mathrm{Bi}_{3}$ is experimentally unknown, so we take the available data of $\mathrm{Ba}_{2} \mathrm{BiSb}_{2}[10]$ for reference. The $\mathrm{Bi}(2)_{y}$ calculated on the three approaches (i.e., LDA, PBE and PBESOL) are very close to each other, within absolute error of $1 \%$ relative to the available experimental data. So is the $\mathrm{Ba}_{z}$. The above analysis indicates the rationality of the constructed lattice model, which implies that the precision of calculations is reliable. The reasonable lattice model should produce the reasonable results of properties. Moreover, a series of lattice constants are set to obtain the total energy $E$ and the corresponding unit cell volume $V$ through the three approaches. Then the obtained $E-V$ data are fitted to the second order Birch-Murnaghan equation of state (EOS) [20]. The zero-pressure bulk modulus $B_{0}$ together with its pressure derivative $B_{0}{ }^{\prime}$ is shown in Table 1. 
Table 1. The equilibrium structural parameters for $\mathrm{Ba}_{2} \mathrm{Bi}_{3}$ obtained from Local Density Approximation (LDA) and General Gradient Approximation (PBE and PBESOL) calculations, including the lattice parameters ( $a, b$ and $c$ in $\AA$ ), the internal parameter $\mathrm{Ba}_{z}$ and $\mathrm{Bi}(2)_{y}$, the equilibrium unit cell volume $\left(V\right.$ in $\left.\AA^{3}\right)$, zero-pressure bulk modulus $\left(B_{0}\right.$ in GPa) and its pressure derivative $\left(B_{0}{ }^{\prime}\right)$ at $0 \mathrm{GPa}$ and $0 \mathrm{~K}$, together with available experimental results.

\begin{tabular}{lllllllllll}
\hline & $a$ & $b$ & $c$ & $b / a$ & $c / a$ & $\mathrm{Ba}_{z}$ & $\mathrm{Bi}(2)_{y}$ & $V$ & $B_{0}$ & $B_{0}{ }^{\prime}$ \\
\hline LDA & 4.7113 & 7.7238 & 9.9284 & 1.6394 & 2.1074 & 0.2778 & 0.2944 & 361.3 & 40.1 & 4.368 \\
PBE & 4.8475 & 7.9389 & 10.2293 & 1.6377 & 2.1102 & 0.2777 & 0.2944 & 393.7 & 31.9 & 4.333 \\
PBESOL & 4.7641 & 7.7862 & 10.0480 & 1.6343 & 2.1091 & 0.2779 & 0.2942 & 372.7 & 36.0 & 4.471 \\
Exp. $^{\mathrm{a}}$ & 4.813 & 7.994 & 9.971 & 1.6609 & 2.0717 & - & - & 383.6 & - & - \\
Exp. $^{\mathrm{b}}$ & 4.819 & 7.989 & 9.982 & 1.6578 & 2.0714 & - & 0.2968 & 384.3 & - & - \\
$\mathrm{Ba}_{2} \mathrm{BiSb}_{2}{ }^{\mathrm{c}}$ & 4.8065 & 7.6721 & 9.8971 & 1.5962 & 2.0591 & 0.2804 & 0.2989 & 365.0 & - & - \\
\hline
\end{tabular}

${ }^{\mathrm{a}}$ Ref. [5]; ${ }^{\mathrm{b}}$ Ref. [7]; ${ }^{\mathrm{c}}$ Ref. [10].

In order to show the pressure effect on the structural parameters of the $\mathrm{Ba}_{2} \mathrm{Bi}_{3}$, the pressure dependence of the ratio $b / a, c / a$, and the normalized $a / a_{0}, b / b_{0}$ and $c / c_{0}$ as well as $V / V_{0}$ (where $a, b, c$ and $V$ are the cell parameters under pressure, $a_{0}, b_{0}, c_{0}$ and $V_{0}$ are the equilibrium cell parameters at zero pressure [5]) are shown in Fig. 2. It is found that the normalized parameters $a / a_{0}, b / b_{0}, c / c_{0}$ and normalized volume $V / V_{0}$ decrease almost linearly with the pressure. The normalized volume $V / V_{0}$ is more sensitive to the pressure. It also can be found that the absolute slope of $c / c_{0}$ is the biggest one, indicating the biggest compressibility along the $c$-axis. When the applied pressure is up to $14 \mathrm{GPa}$, the value of $a, b, c$ and $V$ decreases about $5.85 \%, 8.25 \%$, $9.57 \%$ and $21.89 \%$, respectively. Such phenomenon indicates that the bonding among $\mathrm{Bi}$ ribbon nets is stronger than that among inter-layers. In addition, the ratios of $b / a$ and $c / a$ gradually decrease in a limited variation, implying a pressure isotropic structure of the orthorhombic $\mathrm{Ba}_{2} \mathrm{Bi}_{3}$. Especially, no abrupt change of the $b / a$ and $c / a$ indicates no phase transition, implying the structural stability of the $\mathrm{Ba}_{2} \mathrm{Bi}_{3}$ within the pressure range considered here. 


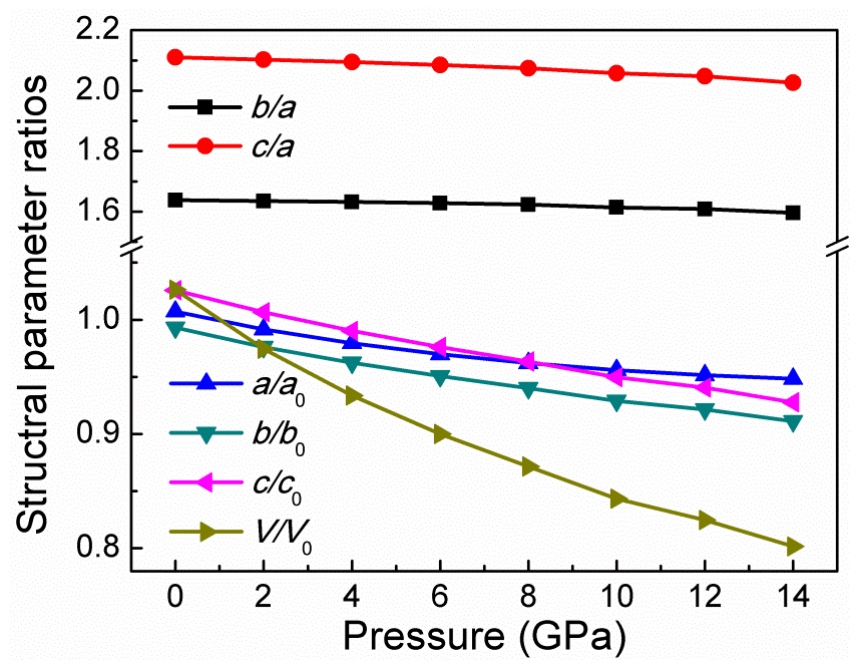

Fig. 2. The structural ratios of $b / a, c / a, a / a_{0}, b / b_{0}, c / c_{0}$ and $V / V_{0}$ of the $\mathrm{Ba}_{2} \mathrm{Bi}_{3}$ as a function of pressure within PBE calculations, where $a_{0}, b_{0}, c_{0}$ and $V_{0}$ are the zero pressure equilibrium structural parameters [5].

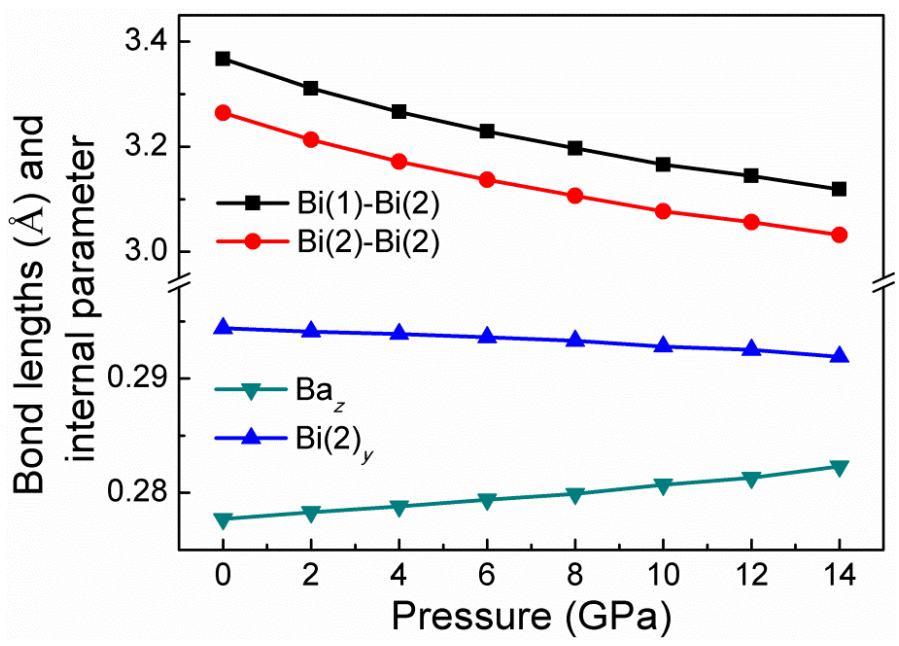

Fig. 3. Variation of bonds length along (001) plane of $\mathrm{Ba}_{2} \mathrm{Bi}_{3}$ and internal parameter $\left(\mathrm{Ba}_{z}\right.$ and $\left.\operatorname{Bi}(2)_{y}\right)$ as a function of pressure within PBE calculations.

In order to know about the underlying reasons of the pressure effect on the structural parameters and volume change of the $\mathrm{Ba}_{2} \mathrm{Bi}_{3}$, the atom bonds length and internal coordinates in the cell are discussed, as shown in Fig. 3. It can be seen that the two bonds length decrease gradually with the pressure, which results in the compression of Bi planes. When the pressure is up to $14 \mathrm{GPa}$, the bonds length of the $\operatorname{Bi}(1)-\operatorname{Bi}(2)$ and $\operatorname{Bi}(2)-\operatorname{Bi}(2)$ is shortened by about $7.37 \%$ and $7.12 \%$, respectively. The similar gradient of the variation of the two bonds length induces the insensitive 
variation of the $\operatorname{Bi}(2)_{y}$ (within the variation of $0.85 \%$ ) to the pressure. On the contrary, the variation of $\mathrm{Ba}_{z}$ is more sensitive to the pressure, indicating the decrease of the distance among layers, which leads to the compression of $c$-axis.

\subsection{Mechanical properties}

Elastic constants are related to elastic moduli, structural stability and anisotropy which play important roles in understanding the nature of materials. Elastic constants can be derived by means of stress-strain approach [21-23] on the basis of Hooke's law [24], in which the stress $(\sigma)$ and strain $(\varepsilon)$ for small deformations to a crystal are linearly related by

$$
c_{i j}=\partial \sigma_{i} / \partial \varepsilon_{j} \quad(i, j=1,2, \ldots, 6),
$$

where the $c_{i j}$ is the elastic constant. For the orthorhombic structure of $\mathrm{Ba}_{2} \mathrm{Bi}_{3}, c_{i j}$ can be expressed as

$$
c_{i j}=\left[\begin{array}{cccccc}
c_{11} & c_{12} & c_{13} & 0 & 0 & 0 \\
c_{12} & c_{22} & c_{23} & 0 & 0 & 0 \\
c_{13} & c_{23} & c_{33} & 0 & 0 & 0 \\
0 & 0 & 0 & c_{44} & 0 & 0 \\
0 & 0 & 0 & 0 & c_{55} & 0 \\
0 & 0 & 0 & 0 & 0 & c_{66}
\end{array}\right] .
$$

It has nine independent elastic constants (i.e., $c_{11}, c_{22}, c_{33}, c_{44}, c_{55}, c_{66}, c_{12}, c_{13}$ and $c_{23}$ ) for the orthorhombic $\mathrm{Ba}_{2} \mathrm{Bi}_{3}$. Additionally, for an orthorhombic structure, the nine independent elastic constants under pressure should satisfy the following mechanical stability conditions [25-27]

$$
\begin{gathered}
\tilde{c}_{11}>0, \tilde{c}_{22}>0, \quad \tilde{c}_{33}>0, \tilde{c}_{44}>0, \quad \tilde{c}_{55}>0, \quad \tilde{c}_{66}>0, \\
\Delta_{1}=\left[\tilde{c}_{11}+\tilde{c}_{22}+\tilde{c}_{33}+2\left(\tilde{c}_{12}+\tilde{c}_{13}+\tilde{c}_{23}\right)\right]>0, \\
\Delta_{2}=\left[\tilde{c}_{11}+\tilde{c}_{22}-2 \tilde{c}_{12}\right]>0, \\
\Delta_{3}=\left[\tilde{c}_{11}+\tilde{c}_{33}-2 \tilde{c}_{13}\right]>0, \\
\Delta_{4}=\left[\tilde{c}_{22}+\tilde{c}_{33}-2 \tilde{c}_{23}\right]>0 .
\end{gathered}
$$


Here the $\tilde{c}_{i i}=c_{i i}-P(\mathrm{i}=1,2, \ldots, 6), \quad \tilde{c}_{12}=c_{12}+P, \tilde{c}_{13}=c_{13}+P, \tilde{c}_{23}=c_{23}+P$, and $P$ is the applied pressure. When the pressure is higher than $14 \mathrm{GPa}$, one of the above mechanical stability criterions, $\Delta_{2}>0$, i.e., Eq. (3c), is no longer fulfilled on the data from the PBE calculations. Such result indicates that the orthorhombic structure of the $\mathrm{Ba}_{2} \mathrm{Bi}_{3}$ is not mechanically stable at the pressures above $14 \mathrm{GPa}$. In order to ensure the reliable results of the $\mathrm{Ba}_{2} \mathrm{Bi}_{3}$ with orthorhombic structure, the investigations under the pressures above $14 \mathrm{GPa}$ are beyond this work.

The calculated elastic constants at $0 \mathrm{GPa}$ are shown in Table 2. The results from LDA, PBE and PBESOL are generally consistent. The elastic constants on the PBE are smaller than that on the other two, which is due to the larger lattice constants calculated on the PBE. The pressure effect on the elastic constants is shown in Fig. 4. It is clearly seen that all independent elastic constants increase monotonously with the pressure.

Table 2. The independent elastic constants ( $c_{i j}$ in $\mathrm{GPa}$ ) of $\mathrm{Ba}_{2} \mathrm{Bi}_{3}$ calculated on the LDA, PBE and PBESOL at $0 \mathrm{GPa}$ and $0 \mathrm{~K}$.

\begin{tabular}{llllllllll}
\hline & $c_{11}$ & $c_{22}$ & $c_{33}$ & $c_{44}$ & $c_{55}$ & $c_{66}$ & $c_{12}$ & $c_{13}$ & $c_{23}$ \\
\hline LDA & 60.6 & 70.6 & 73.3 & 20.1 & 27.7 & 25.0 & 38.8 & 25.8 & 16.2 \\
PBE & 49.3 & 59.3 & 63.6 & 14.8 & 20.9 & 19.3 & 32.7 & 19.4 & 11.7 \\
PBESOL & 55.4 & 64.5 & 68.6 & 18.0 & 25.0 & 21.9 & 35.9 & 23.0 & 13.9 \\
\hline
\end{tabular}

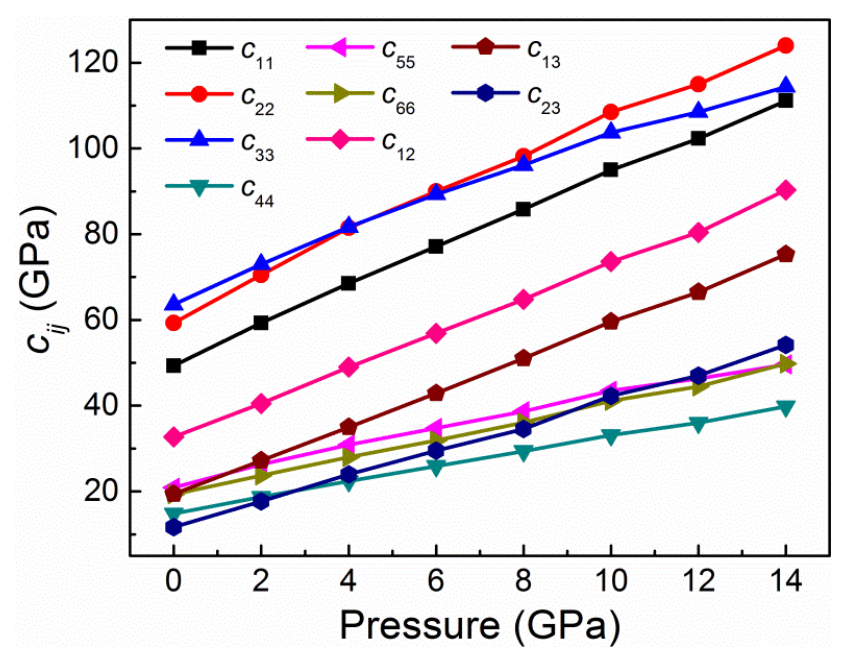

Fig. 4. Pressure dependence of the elastic constants $\left(c_{i j}\right)$ of $\mathrm{Ba}_{2} \mathrm{Bi}_{3}$ within PBE calculations. 
Generally, according to Voigt-Ruess-Hill approximations [28], the polycrystalline elastic moduli can be obtained based on the results of elastic constants of single crystal. For orthorhombic structure, the Voigt and Reuss bulk modulus $\left(B_{V}\right.$, $\left.B_{R}\right)$, Voigt and Reuss shear modulus $\left(G_{V}, G_{R}\right)$ can be given as $[29,30]$

$$
\begin{gathered}
B_{V}=\frac{1}{9}\left[\left(c_{11}+c_{22}+c_{33}\right)+2\left(c_{12}+c_{13}+c_{23}\right)\right], \\
B_{R}=\left[\left(s_{11}+s_{22}+s_{33}\right)+2\left(s_{12}+s_{13}+s_{23}\right)\right]^{-1}, \\
G_{V}=\frac{1}{15}\left[\left(c_{11}+c_{22}+c_{33}\right)-\left(c_{12}+c_{13}+c_{23}\right)+3\left(c_{44}+c_{55}+c_{66}\right)\right], \\
G_{R}=15\left[4\left(s_{11}+s_{22}+s_{33}\right)-4\left(s_{12}+s_{13}+s_{23}\right)+3\left(s_{44}+s_{55}+s_{66}\right)\right]^{-1},
\end{gathered}
$$

where the $s_{i j}$ are the elastic compliance constants (i.e., $s_{i j}=c_{i j}^{-1}$ ). Finally, the elastic moduli of the polycrystalline can be approximated by Hill's average, for bulk modulus $\left.B=\left(B_{V}+B_{R}\right) / 2\right)$, and for shear modulus $G=\left(G_{V}+G_{R}\right) / 2$. Further, the Young's modulus $(E)$ and Poisson's ratio ( $v$ ) are given by

$$
E=\frac{9 B G}{3 B+G} \text { and } v=\frac{3 B-2 G}{2(3 B+G)} .
$$

The above mentioned elastic moduli and Poisson's ratio for the $\mathrm{Ba}_{2} \mathrm{Bi}_{3}$ at $0 \mathrm{GPa}$ are shown in Table 3. The results from the three approaches are generally consistent with each other, attributed to the consistent elastic constants. Specially, the bulk modulus calculated from elastic constants is in good agreement with that from second order Birch-Murnaghan equation of state fitting (as listed in Table 1), which means that the present calculations are reliable. It is also found from Table 3 that the bulk modulus is as small as that in other layered structures [31], indicating good compressibility of the orthorhombic $\mathrm{Ba}_{2} \mathrm{Bi}_{3}$. The polycrystalline elastic moduli (including bulk modulus $B$, shear modulus $G$ and Young's modulus $E$ ) of the $\mathrm{Ba}_{2} \mathrm{Bi}_{3}$ as a function of pressure are shown in Fig. 5. It is obviously seen that the elastic moduli are significantly influenced by the external pressure and monotonously increase with the pressure. 
Table 3. The bulk modulus ( $B$ in GPa) and shear modulus ( $G$ in GPa) for polycrystalline $\mathrm{Ba}_{2} \mathrm{Bi}_{3}$ obtained from the elastic constants using Voigt-Reuss-Hill approximations. The Young's modulus ( $E$ in Gpa), Poisson's ratio $(v)$ and $G / B$ are estimated from Hill's approximation at $0 \mathrm{GPa}$ and $0 \mathrm{~K}$.

\begin{tabular}{llllllllll}
\hline & $B_{R}$ & $B_{V}$ & $B$ & $G_{R}$ & $G_{V}$ & $G$ & $E$ & $G / B$ & $v$ \\
\hline LDA & 40.6 & 40.7 & 40.7 & 20.7 & 22.9 & 21.8 & 55.5 & 0.536 & 0.273 \\
PBE & 33.2 & 33.3 & 33.3 & 16.3 & 18.2 & 17.3 & 44.2 & 0.519 & 0.279 \\
PBESOL & 37.0 & 37.1 & 37.1 & 18.6 & 20.7 & 19.7 & 50.2 & 0.531 & 0.274 \\
\hline
\end{tabular}

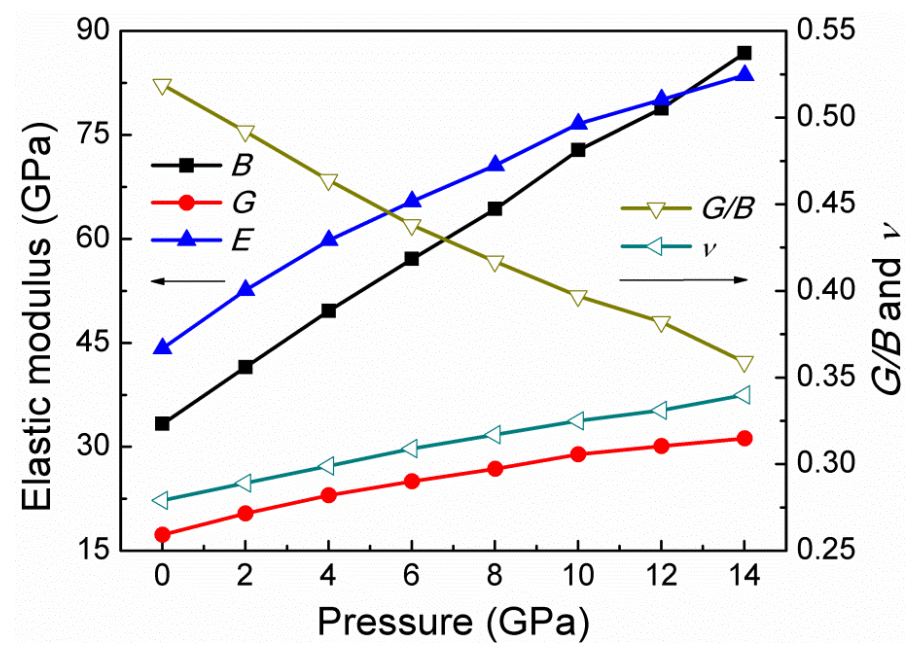

Fig. 5. Pressure dependence of elastic moduli $(B, G$ and $E)$ and ductility factor $(G / B$ and Poisson ratio v) within PBE calculations.

Additionally, the ductility and brittleness play a vital role in the material manufacturing. The ductility and brittleness are usually estimated through two ductility factors, i.e., Poisson's ratio $(v)$ and ratio of $G / B$. According to Frantsevich's rule [32], set $1 / 3$ as the critical value of Poisson's ratio. If $v$ is less than $1 / 3$, the material is dominated by brittleness, otherwise, ductility. For orthorhombic $\mathrm{Ba}_{2} \mathrm{Bi}_{3}$, the value of $v$ is around 0.27 (see Table 3), smaller than $1 / 3$, which means that the orthorhombic $\mathrm{Ba}_{2} \mathrm{Bi}_{3}$ is a brittle material. On the other hand, Pugh [33] presented a simple relationship: a high (low) value of $G / B$ is associated with brittleness (ductility), with the critical value of 0.571 . In this work, the value of $G / B$ is around 0.53 (see Table 3), smaller than 0.571 , indicating a ductile manner of the $\mathrm{Ba}_{2} \mathrm{Bi}_{3}$. Given the fact 
that the results of ductility from the two rules are contradictory, we can conclude that the $\mathrm{Ba}_{2} \mathrm{Bi}_{3}$ lies in between the brittle and ductile border line at $0 \mathrm{GPa}$. The pressure effect on the ductility of the $\mathrm{Ba}_{2} \mathrm{Bi}_{3}$ is shown in Fig. 5. The conclusion from the variation of Poisson's ratio $v$ is consistent with that from the variation of $G / B$. Consequently, for the orthorhombic $\mathrm{Ba}_{2} \mathrm{Bi}_{3}$, the pressure can enhance the ductility.

\subsection{Elastic anisotropy}

The usually used anisotropic factors are shear anisotropic factors $\left(A_{1}, A_{2}\right.$ and $\left.A_{3}\right)$, the directional bulk modulus along different crystallographic axis $\left(B_{a}, B_{b}\right.$ and $\left.B_{c}\right)$, compressibility anisotropic factors $\left(A_{B b}\right.$ and $\left.A_{B c}\right)$, percent anisotropies $\left(A_{G}\right.$ and $\left.A_{B}\right)$ and universal anisotropic index $\left(A^{U}\right)$. All the results of the anisotropy of $\mathrm{Ba}_{2} \mathrm{Bi}_{3}$ obtained from LDA, PBE and PBESOL at $0 \mathrm{GPa}$ are shown in Table 4.

Table 4. The shear anisotropic factors $\left(A_{1}, A_{2}\right.$ and $\left.A_{3}\right)$, the directional bulk modulus along different crystallographic axis $\left(B_{a}, B_{b}\right.$ and $B_{c}$ in GPa), compressibility anisotropic factors $\left(A_{B b}\right.$ and $\left.A_{B c}\right)$, percent anisotropies $\left(A_{G}\right.$ and $A_{B}$ in $\left.\%\right)$ and universal anisotropic index $\left(A^{U}\right)$ of $\mathrm{Ba}_{2} \mathrm{Bi}_{3}$ obtained from LDA, PBE and PBESOL calculations at $0 \mathrm{GPa}$ and $0 \mathrm{~K}$.

\begin{tabular}{llllllllllll}
\hline & $A_{1}$ & $A_{2}$ & $A_{3}$ & $B_{a}$ & $B_{b}$ & $B_{c}$ & $A_{B b}$ & $A_{B c}$ & $A_{G}$ & $A_{B}$ & $A^{U}$ \\
\hline LDA & 0.977 & 0.994 & 1.866 & 133.6 & 126.2 & 108.0 & 0.944 & 0.809 & 5.046 & 0.123 & 0.534 \\
PBE & 0.799 & 0.840 & 1.787 & 102.3 & 107.6 & 90.7 & 1.052 & 0.886 & 5.495 & 0.138 & 0.584 \\
PBESOL & 0.923 & 0.950 & 1.821 & 122.1 & 113.9 & 99.5 & 0.932 & 0.814 & 5.344 & 0.135 & 0.567 \\
\hline
\end{tabular}

The shear anisotropic factors provide a measure of the degree of anisotropy in the bonding between atoms in different planes [29]. The shear anisotropic factor for $\{100\}$ shear plane between the $<011>$ and $<010>$ direction is

$$
A_{1}=\frac{4 c_{44}}{c_{11}+c_{33}-2 c_{13}}
$$

That for the $\{010\}$ shear planes between $<101>$ and $<001>$ direction is

$$
A_{2}=\frac{4 c_{55}}{c_{22}+c_{33}-2 c_{23}}
$$

And that for the $\{001\}$ shear planes between $\langle 110\rangle$ and $\langle 010\rangle$ direction is 


$$
A_{3}=\frac{4 c_{66}}{c_{11}+c_{22}-2 c_{12}} \text {. }
$$

On the other hand, for orthorhombic structure, the shear anisotropic factors are not sufficient to describe the elastic anisotropy. The anisotropy of linear bulk modulus is also considered. The directional bulk modulus along different crystallographic axis can be defined as $B_{i}=i(d P / d i)(i=a, b$ and $c)[29,34]$. The compressibility anisotropies of bulk modulus along the $b$ axis and $c$ axis with respect to the $a$ axis can be written as

$$
A_{B b}=\frac{B_{b}}{B_{a}} \text { and } A_{B c}=\frac{B_{c}}{B_{a}} .
$$

Note that both for the shear and compressibility anisotropic factors, the numerical one indicates elastic isotropy and any departure from one corresponds to a degree of elastic anisotropy [29].

The results of shear anisotropy indicate that the orthorhombic $\mathrm{Ba}_{2} \mathrm{Bi}_{3}$ is nearly shear isotropic in $\{100\}$ and $\{010\}$ planes, which can be attributed to the directional bonding between $\mathrm{Bi}$ atoms in $a b$ plane that is close to honeycomb. While the elastic anisotropy in $\{001\}$ shear planes between $\langle 110\rangle$ and $\langle 010\rangle$ is larger than that in the other two, which can be attributed to the two-dimensional layered structure along the $c$ axis. These results reveal that the $\{001\}$ shear plane are much easier to be the cleavage plane among these principal planes. Moreover, compared to $B_{a}$ and $B_{b}$, the directional bulk modulus $B_{c}$ along $c$ axis is the smallest, which explains the phenomenon that $c$ axis is more compressible. The compressibility anisotropic factors ( $A_{B b}$ and $A_{B c}$ ) are close to one, indicating that the orthorhombic $\mathrm{Ba}_{2} \mathrm{Bi}_{3}$ is nearly bulk isotropic. The pressure dependence of the shear anisotropic factors and compressibility anisotropic factors are shown in Fig. 6(a). It is obvious that the deviations from one are getting larger with the pressure, indicating more anisotropic for the $\mathrm{Ba}_{2} \mathrm{Bi}_{3}$. 

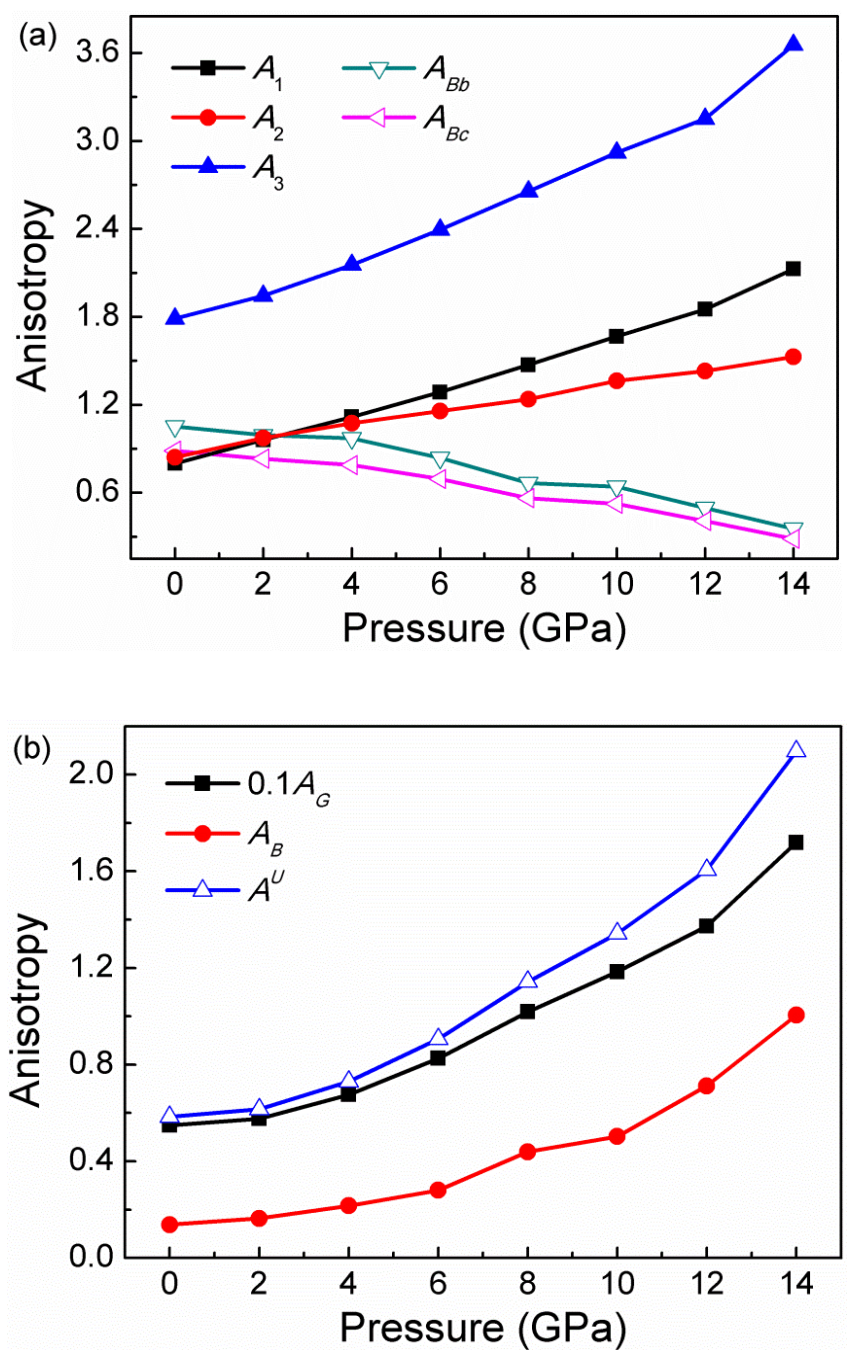

Fig. 6. Pressure dependence of anisotropy factors of $\mathrm{Ba}_{2} \mathrm{Bi}_{3}$ obtained from PBE calculations, including (a) the shear anisotropic factors $\left(A_{1}, A_{2}\right.$ and $\left.A_{3}\right)$, compressibility anisotropy factors $\left(A_{B b}\right.$ and $\left.A_{B c}\right)$, (b) percent anisotropies $\left(A_{G}\right.$ and $A_{B}$, in \%) and universal anisotropic index $\left(A^{U}\right)$.

In addition, the percentage anisotropy in compressibility and shear is also under consideration, defined as

$$
A_{B}=\frac{B_{V}-B_{R}}{B_{V}+B_{R}} \text { and } A_{G}=\frac{G_{V}-G_{R}}{G_{V}+G_{R}} .
$$

For these two expressions, a value of zero represents elastic isotropy and a value of $100 \%$ is the largest possible anisotropy [35]. Alternatively, the universal anisotropic index is employed, which is defined as

$$
A^{U}=5 \frac{G_{V}}{G_{R}}+\frac{B_{V}}{B_{R}}-6 \geq 0,
$$


where $A^{U}=0$ is for isotropic materials and the departure from zero defines the extent of anisotropy [36]. As shown in Table 4, obviously, the universal anisotropy index and percentage of anisotropies are relatively small at $0 \mathrm{GPa}$, implying that the orthorhombic $\mathrm{Ba}_{2} \mathrm{Bi}_{3}$ is nearly isotropic, especially bulk isotropic. The percentage anisotropy and universal anisotropy factors all gradually increase with the pressure, as shown in Fig. 6(b), which is consistent with the results of shear and compressibility anisotropic factors.
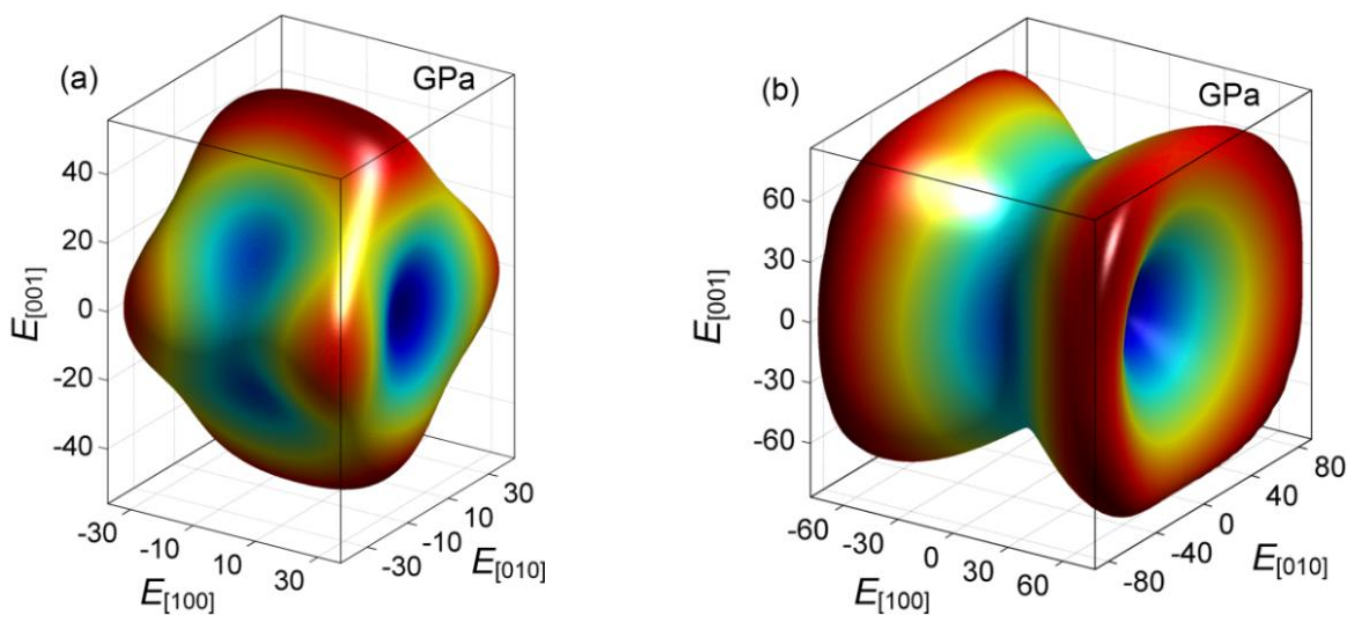

Fig. 7. 3D directional dependence of the Young's modulus (in GPa) of $\mathrm{Ba}_{2} \mathrm{Bi}_{3}$ at (a) $0 \mathrm{GPa}$ and (b) $14 \mathrm{GPa}$ within PBE calculations.

In order to further illustrate the anisotropy of the $\mathrm{Ba}_{2} \mathrm{Bi}_{3}$, a more vivid way is to show the three dimensional (3D) contours of the elastic moduli. The direction dependent Young's modulus $(E)$ for orthorhombic crystals can be defined as $[29,37]$

$$
\frac{1}{E}=s_{11} l_{1}^{4}+\left(2 s_{12}+s_{66}\right) l_{1}^{2} l_{2}^{2}+\left(2 s_{13}+s_{55}\right) l_{1}^{2} l_{3}^{2}+s_{22} l_{2}^{4}+\left(2 s_{23}+\mathrm{s}_{44}\right) l_{2}^{2} l_{3}^{2}+s_{33} l_{3}^{4},
$$

where $s_{i j}$ are the elastic compliance constants, and $l_{1}=\sin \theta \cos \varphi, l_{2}=\sin \theta \sin \varphi$ and $l_{3}=\cos \theta$ are the directional cosines of angles with the three principal directions. The shape of 3D directional dependence of an isotropic system is like a sphere, and the departure from the spherical shape reflects the extent of anisotropy. The 3D surface of the Young's modulus of the $\mathrm{Ba}_{2} \mathrm{Bi}_{3}$ under $0 \mathrm{GPa}$ and $14 \mathrm{GPa}$ are presented in Fig. 7. It can be seen from Fig. 7(a) that 3D surface of the Young's modulus for the $\mathrm{Ba}_{2} \mathrm{Bi}_{3}$ at 0 GPa shows a large deviation from the sphere shape, so we can conclude that the 
$\mathrm{Ba}_{2} \mathrm{Bi}_{3}$ has a character of obvious elastic anisotropy. From Fig. 7(b), we can see that the shape under $14 \mathrm{GPa}$ is in larger deformation, indicating more anisotropic under higher pressures.
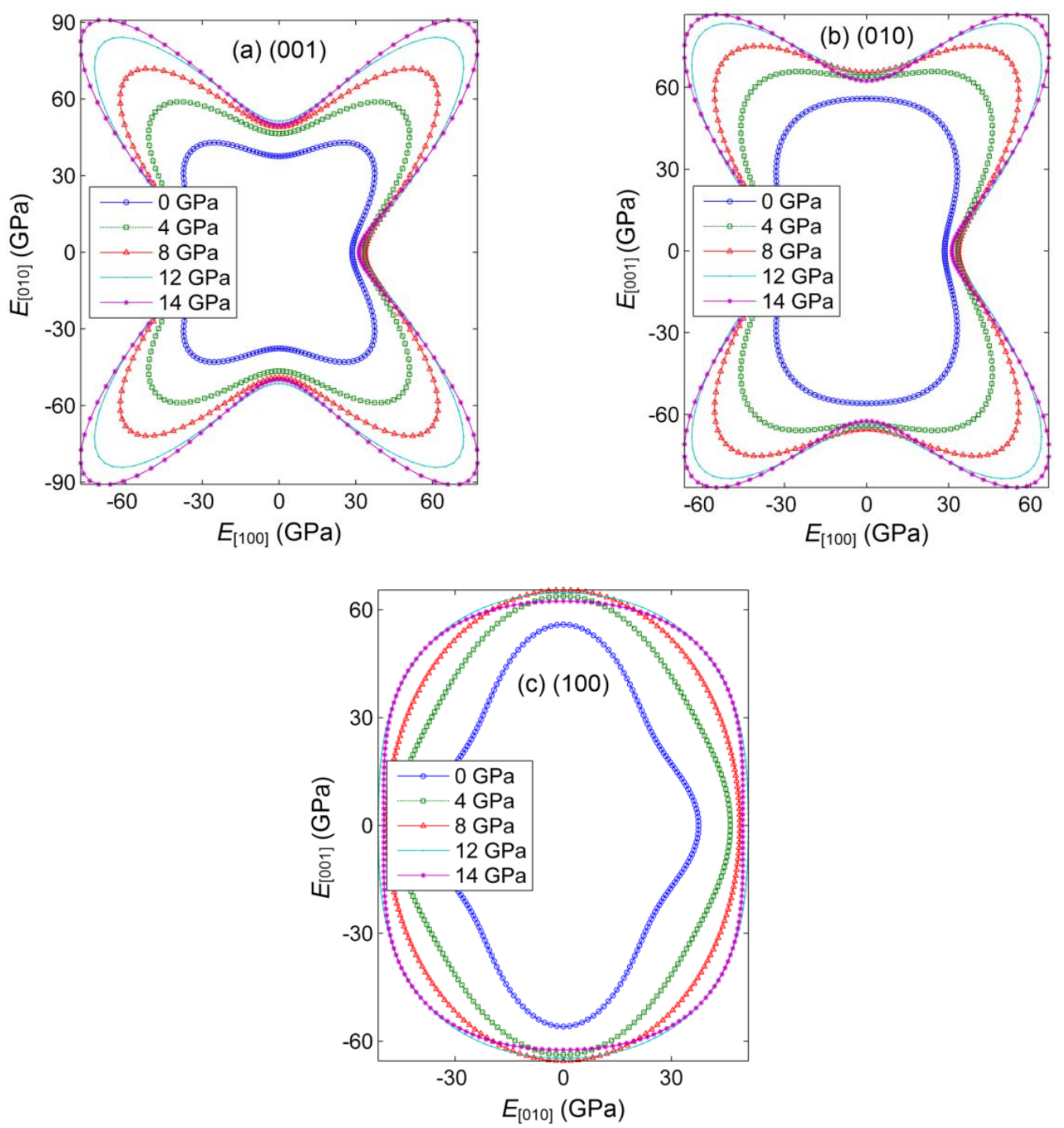

Fig. 8. The planar contours of Young's modulus of $\mathrm{Ba}_{2} \mathrm{Bi}_{3}$ are shown for (a) (001), (b) (010) and (c) (100) crystallographic planes under various pressures within PBE calculations.

For the more comprehensive visualization of the elastic anisotropy, the projections of Young's modulus on (001), (010) and (100) crystallographic planes are depicted in Fig. 8. We can see that the projected Young's modulus on (001) plane shows more anisotropic features than that on the other two planes. The shapes of the projected Young's modulus on (001) plane resemble that on (010) plane under various 
pressures. The anisotropy in these two planes is strengthening with the pressure, while the character in (100) plane tends to be isotropic. These results are in good agreement with the conclusions from the analysis of above mentioned anisotropic factors. It is noted that the results of 3D contours of Young's modulus from LDA and PBESOL are similar to that from PBE, so not shown here.

According to the above analysis of anisotropy, it is finally concluded that the orthorhombic $\mathrm{Ba}_{2} \mathrm{Bi}_{3}$ is nearly isotropic at $0 \mathrm{GPa}$, and the pressure can make the orthorhombic $\mathrm{Ba}_{2} \mathrm{Bi}_{3}$ more anisotropic.

\subsection{Electronic properties}

In previous studies [7, 10], the electronic related calculations were performed without spin-orbit coupling for the $\mathrm{Ba}_{2} \mathrm{Bi}_{3}$ and $\mathrm{Ba}_{2} \mathrm{BiSb}_{2}$. To keep the consistency of the calculations, the spin-orbit coupling is ignored here. The energy band structure of the primitive cell of the $\mathrm{Ba}_{2} \mathrm{Bi}_{3}$ from the FP-LAPW calculations is shown in Fig. 9, similar to that of $\mathrm{Ba}_{2} \mathrm{BiSb}_{2}$ [10]. Though $\mathrm{Ba}_{2} \mathrm{Bi}_{3}$ is literally different from $\mathrm{Ba}_{2} \mathrm{BiSb}_{2}$, the $\mathrm{Ba}_{2} \mathrm{Bi}_{3}$ is the parent phase of $\mathrm{Ba}_{2} \mathrm{BiSb}_{2}$ and also has a similar layered structure as well as chemical constituents to that of $\mathrm{Ba}_{2} \mathrm{BiSb}_{2}$ [10]. So the two intimate compounds can induce similar energy band structures. The similarity indicates the rationality of the electronic related results at $0 \mathrm{GPa}$ in this work. It can be seen from Fig. 9(a) that there are two bands crossing the Fermi level $\left(E_{F}\right)$ along W-R, R- $\Gamma$ and $\Gamma$-X symmetry directions, indicating metallic character (such as metallic electrical and thermal conductivity) of the $\mathrm{Ba}_{2} \mathrm{Bi}_{3}$. For the energy band structure at $14 \mathrm{GPa}$, as shown in Fig. 9(b), there is only one band crossing the Fermi level, which indicates less occupied states at Fermi level. Such difference can be attributed to the fact that the pressure makes the Fermi energy higher. Moreover, the extent of energy dispersion gets larger, especially along W-R direction, which means that the local character of electrons gets weaker and the itinerant character becomes stronger at 14 GPa. 


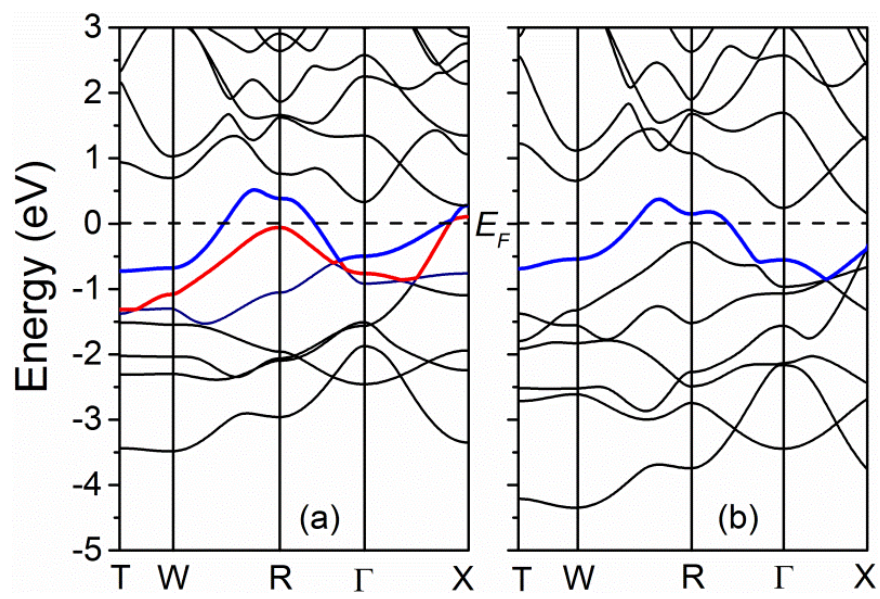

Fig. 9. Electronic band structures of the primitive cell of $\mathrm{Ba}_{2} \mathrm{Bi}_{3}$ along the high symmetry direction around the Fermi level $\left(E_{F}\right)$ calculated on the PBE: (a) 0 GPa, and (b) $14 \mathrm{GPa}$. In both (a) and (b) the Fermi level is set to be zero, and indicated by the horizontal dotted line. The thick lines (not black) represent bands crossing the Fermi level.

In Fig. 10, we show the electronic total density of states (DOS), together with the partial DOS of atom $\mathrm{Ba}$ and $\mathrm{Bi}$ of the $\mathrm{Ba}_{2} \mathrm{Bi}_{3}$, which should help to further understand the energy band structure and gain a deep insight into the bonding characteristics. As shown in Fig. 10(a), the total DOS of the $\mathrm{Ba}_{2} \mathrm{Bi}_{3}$ exhibits non-zero value at $E_{F}$, indicating metallic character, which is consistent with the results of energy band structure. From Fig. 10, together with Fig. 9, one can find that the bands from $-5 \mathrm{eV}$ to $E_{F}$ are mainly contributed by $\mathrm{Ba}-d$ and $\mathrm{Bi}-p$ states and basically the contributions from other states can be neglected. In addition, there is a deep valley near the Fermi level (i.e., pseudo energy gap) in the DOS of the $\mathrm{Ba}_{2} \mathrm{Bi}_{3}$, which indicates that there exists a strong hybridization in the $\mathrm{Ba}_{2} \mathrm{Bi}_{3}$ crystal. It can be seen that $\mathrm{Ba}-d$ orbit hybridizes strongly with $\mathrm{Bi}-p$ orbit. The hybridization peaks around $E_{F}$, typically at the energy of $-1.38 \mathrm{eV},-1.10 \mathrm{eV}$ and $-0.20 \mathrm{eV}$, indicate the presence of covalent bonds between atoms $\mathrm{Ba}$ and $\mathrm{Bi}$. Compared to the DOS at $0 \mathrm{GPa}$, the total and partial DOS show slight changes at $14 \mathrm{GPa}$, indicating that the orthorhombic $\mathrm{Ba}_{2} \mathrm{Bi}_{3}$ still keeps structural stability under the pressures up to $14 \mathrm{GPa}$. The hybridization effects at 14 $\mathrm{GPa}$ are identical to that at $0 \mathrm{GPa}$. Moreover, the height of the hybridization peak gets lower, implying weaker hybridization between $\mathrm{Ba}-d$ and Bi-p orbits at $14 \mathrm{GPa}$. The bands are broadened, implying stronger delocalization under higher pressures. 

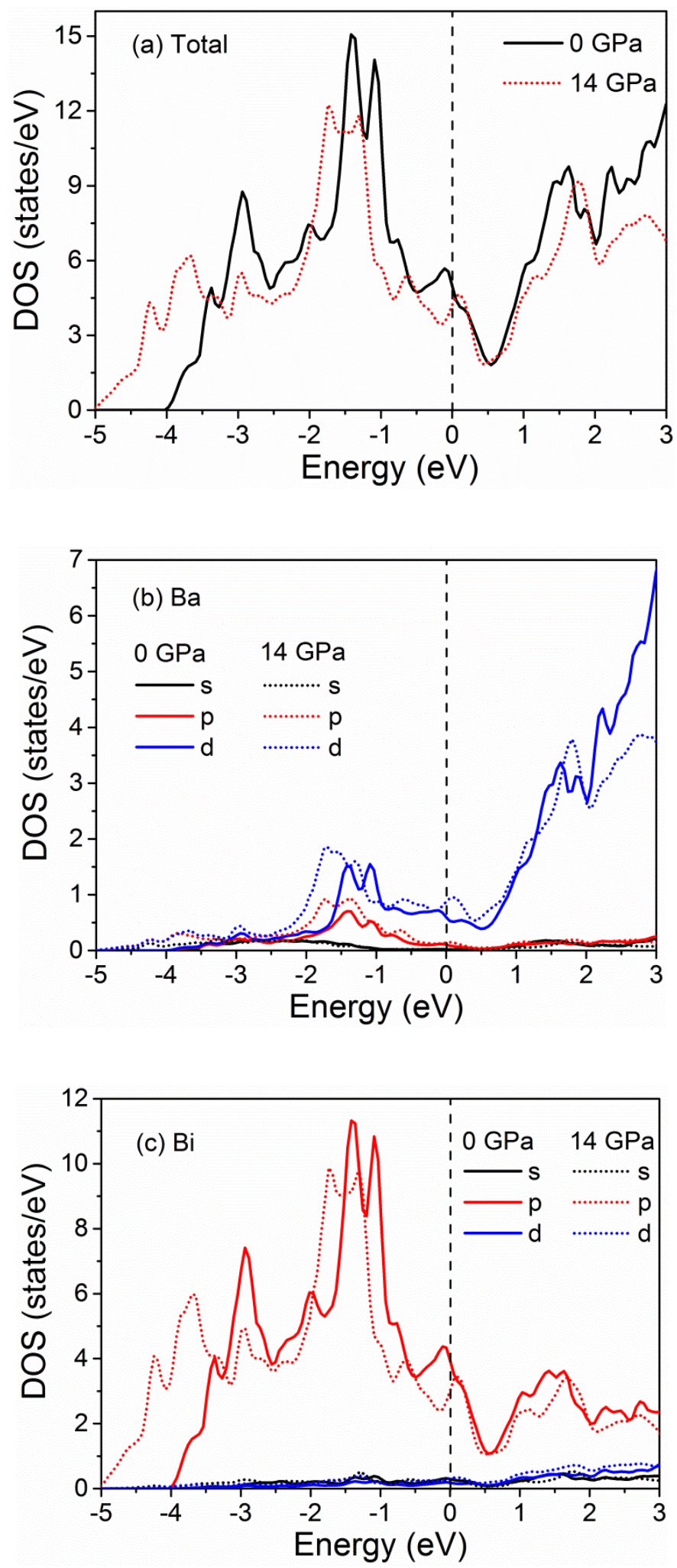

Fig. 10. (a) The total density of states (DOS, in states/eV per unit cell), (b) the partial DOS of atom $\mathrm{Ba}$ and (c) of atom $\mathrm{Bi}$ of the $\mathrm{Ba}_{2} \mathrm{Bi}_{3}$ under the pressure $0 \mathrm{GPa}$ (solid line) and $14 \mathrm{GPa}$ (dot) within PBE calculations. Here the Fermi level is indicated by the vertical dotted line. 
The total DOS at $E_{F}\left(N\left(E_{F}\right)\right)$ of the $\mathrm{Ba}_{2} \mathrm{Bi}_{3}$ is 5.0549 states/eV per unit cell at 0 GPa on PBE calculations, consistent with the result of about 4.5 states/eV per unit cell (TB-LMTO-ASA method) [7]. The different results of $N\left(E_{F}\right)$ should arise from the different methods. The individual contributions to the total $N\left(E_{F}\right)$ at $0 \mathrm{GPa}$ and 14 GPa are collected in Table 5. The contributions of atoms $\mathrm{Ba}$ and $\mathrm{Bi}$ to the total $N\left(E_{F}\right)$ are as much as $14.07 \%$ and $85.93 \%$, respectively. The results are in good accordance with that of about $13 \%$ and $87 \%$ [7]. Such consistency further supports the validity of the lattice structures as well as the rationality of the electronic related results. It is noted that the reference results of the $N\left(E_{F}\right)$ are roughly estimated according to the "Figure 3(a)" in Ref. [7]. Especially, the Ba- $d$ and Bi- $p$ states contribute $11.61 \%$ and $76.70 \%$, respectively. Thus, one can explicitly conclude that the electronic properties of the $\mathrm{Ba}_{2} \mathrm{Bi}_{3}$ are greatly influenced by Ba- $d$ and $\mathrm{Bi}-p$ states that are strongly involved in the formation of the superconductivity. When the pressure increases up to $14 \mathrm{GPa}$, the contributions from Ba- $p, \mathrm{Ba}-d$ and $\mathrm{Bi}-d$ states at $E_{F}$ show a slight increase, but the total $N\left(E_{F}\right)$ decreases. The pressure dependence of the total $N\left(E_{F}\right)$ of the $\mathrm{Ba}_{2} \mathrm{Bi}_{3}$ is shown in Fig. 11. It can be seen that the total $N\left(E_{F}\right)$ decreases rapidly under the pressures from zero to $4 \mathrm{GPa}$, varies a little (less 1.8\%) from $4 \mathrm{GPa}$ to $12 \mathrm{GPa}$ and then decreases rapidly under the pressure up to $14 \mathrm{GPa}$. Such phenomenon may be attributed to that the DOS of the $\mathrm{Ba}_{2} \mathrm{Bi}_{3}$ is sensitive to the lattice structure. The relationship between them satisfy the following equation

$$
N\left(E_{F}\right)=5.06754-0.27676 P+0.03532 P^{2}-0.0014 P^{3} .
$$

Here, the unit of total $N\left(E_{F}\right)$ is states/eV per unit cell, and the unit of pressure $(P)$ is GPa. As a whole, the total $N\left(E_{F}\right)$ of the $\mathrm{Ba}_{2} \mathrm{Bi}_{3}$ decreases with the pressure among 0 14 GPa.

Table 5. Density of states (states/eV) per unit cell at Fermi level of the $\mathrm{Ba}_{2} \mathrm{Bi}_{3}$ under the pressure $0 \mathrm{GPa}$ and $14 \mathrm{GPa}$ at $0 \mathrm{~K}$ within PBE calculations.

\begin{tabular}{cccccccc}
\hline & \multicolumn{3}{c}{$0 \mathrm{GPa}$} & & \multicolumn{3}{c}{$14 \mathrm{GPa}$} \\
\cline { 2 - 4 } \cline { 6 - 7 } Atoms & $\mathrm{s}$ & $\mathrm{p}$ & $\mathrm{d}$ & & $\mathrm{s}$ & $\mathrm{p}$ & $\mathrm{d}$ \\
\hline $\mathrm{Ba}$ & 0.0205 & 0.1064 & 0.5973 & & 0.0201 & 0.1417 & 0.8734 \\
$\mathrm{Bi}$ & 0.2940 & 3.9472 & 0.1807 & & 0.2589 & 3.0180 & 0.3179 \\
\hline
\end{tabular}




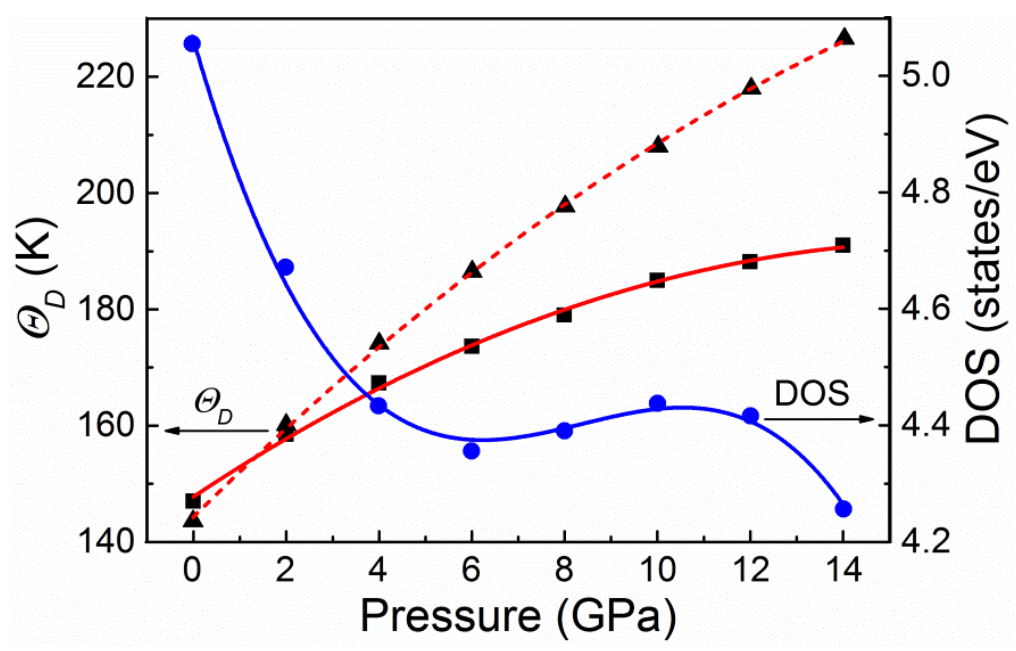

Fig. 11. The pressure dependence of total density of states (DOS) at Fermi level and Debye temperature $\left(\Theta_{D}\right)$ of the $\mathrm{Ba}_{2} \mathrm{Bi}_{3}$ within PBE calculations. The blue solid line is for DOS. The red dotted line is for $\Theta_{D}$ calculated from quasi-harmonic Debye model. The red solid line is for $\Theta_{D}$ calculated from elastic moduli.

\subsection{Thermodynamic properties}

To investigate the thermodynamic properties of the $\mathrm{Ba}_{2} \mathrm{Bi}_{3}$, the quasi-harmonic Debye model is employed. The method is realized in the GIBBS code [38] that are usually used [34, 39, 40]. The detailed calculation procedure is shown in Ref. [38]. In the GIBBS code, the energy-volume points are used as the input data. In this work, a series of minimum energies of fixed volumes are calculated through optimizing the structural parameters of the corresponding volumes. Here these energy-volume points are calculated by employing Quantum-Espresso package. Then the heat capacity at constant volume $C_{V}$, thermal expansion coefficient $\alpha$ and Grüneisen parameter $\gamma$ as well as Debye temperature $\Theta_{D}$ of the $\mathrm{Ba}_{2} \mathrm{Bi}_{3}$ at various temperatures and pressures are obtained. It's noted that the $\Theta_{D}$ is computed from the static bulk moduli when employing GIBBS code in this work. It's worth mentioning that the $\Theta_{D}$ obtained through GIBBS code plays a vital role in the verification of $\Theta_{D}$ obtained from elastic moduli.

The variations of the heat capacity at constant volume $C_{V}$ of the $\mathrm{Ba}_{2} \mathrm{Bi}_{3}$ as a function of temperature under various pressures are shown in Fig. 12. It can be seen that the $C_{V}$ shows a sharp increase under the tempratures up to $150 \mathrm{~K}$ and increases 
slowly at higher temperature, which is attributed to the anharmonic approximation used in the GIBBS code. In addition, the $C_{V}$ increases with the temperature at a given pressure and decreases with the pressure at a given temperature. The pressure has less influence on the heat capacity than temperature. At higher temperatures $(>150 \mathrm{~K})$, the $C_{V}$ is close to the so-called Dulong-Petit limit [41] $\left(249.3 \mathrm{~J} \cdot \mathrm{mol}^{-1} \cdot \mathrm{K}^{-1}\right.$ for $\left.\mathrm{Ba}_{2} \mathrm{Bi}_{3}\right)$, which is reasonable to all solids at high temperatures.

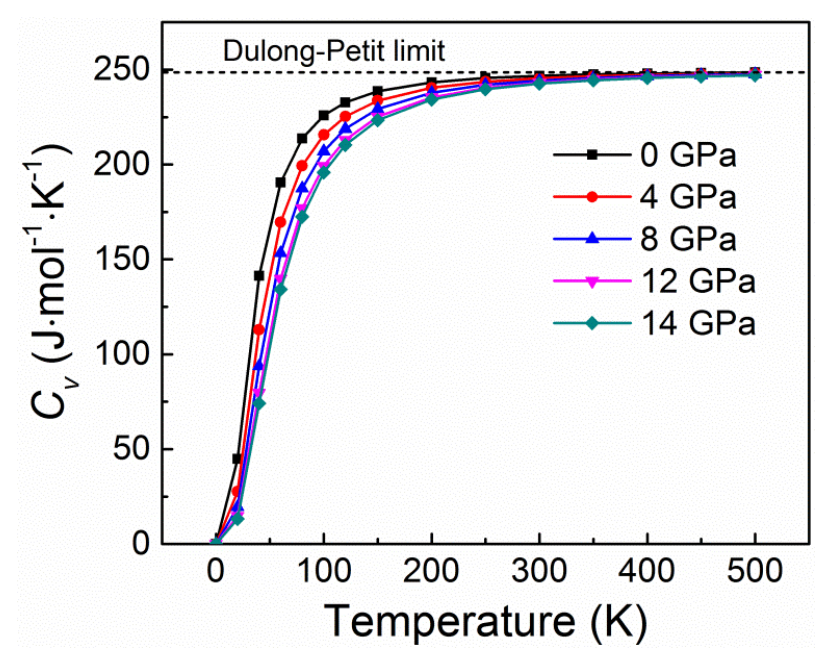

Fig. 12. Relationship between the heat capacity at constant volume $\left(C_{V}\right)$ and temperature of the $\mathrm{Ba}_{2} \mathrm{Bi}_{3}$ under various pressures within $\mathrm{PBE}$ calculations. The horizontal dash line is Dulong-Petit limit that is close to $249.3 \mathrm{~J} \cdot \mathrm{mol}^{-1} \cdot \mathrm{K}^{-1}$ for $\mathrm{Ba}_{2} \mathrm{Bi}_{3}$.

The temperature dependence of the thermal expansion coefficient $\alpha$ of the $\mathrm{Ba}_{2} \mathrm{Bi}_{3}$ under various pressures is shown in Fig. 13. We can see that the thermal expansion coefficient increases rapidly with the temperature and gradually approaches a gently linear increase at high temperatures $(>150 \mathrm{~K})$. The thermal expansion coefficient decreases tempestuously with pressure increasing, indicating that the pressure has significant effect on the thermal expansion coefficient.

The value of $\gamma$ at $0 \mathrm{GPa}$ and $0 \mathrm{~K}$ is listed in Table 6 , with consistent results from LDA, PBE and PBESOL calculations. The variation of Grüneisen parameter $(\gamma)$ with pressure and temperature are displayed in Fig. 14. In Fig. 14(a), it can be seen that the $\gamma$ decreases sharply with pressure increasing at a given temperature. As the pressure is enhanced, the effect of temperature becomes weaker and the four curves are getting 
converged together at higher pressures. Such phenomenon is clearly shown in Fig. 14(b). It is evident that the $\gamma$ varies less at higher pressure. In a word, the pressure has much stronger influence on $\gamma$ than temperature.

Overall, it is concluded that the pressure makes the temperature effect weaker on the heat capacity at constant volume $C_{V}$, thermal expansion coefficient $\alpha$ and Grüneisen parameter $\gamma$ of the $\mathrm{Ba}_{2} \mathrm{Bi}_{3}$. In other words, the pressure and temperature have opposite effect on the three thermodynamic quantities.

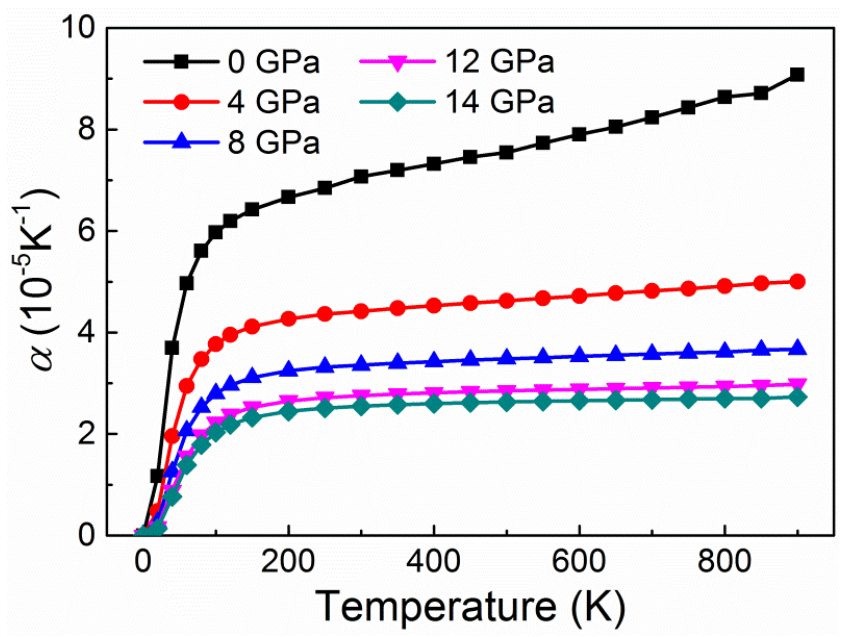

Fig. 13. Relationship between the thermal expansion coefficient $(\alpha)$ and temperature of $\mathrm{Ba}_{2} \mathrm{Bi}_{3}$ under various pressures within PBE calculations.

Table 6 . The density $\left(\rho\right.$ in $\left.\mathrm{g} / \mathrm{cm}^{3}\right)$, transverse, longitudinal, average elastic wave velocity $\left(v_{t}, v_{l}\right.$, $v_{m}$ in $\left.\mathrm{m} / \mathrm{s}\right)$, Debye temperature $\left(\Theta_{D}\right.$ in $\left.\mathrm{K}\right)$ from the average elastic wave velocity obtained from polycrystalline elastic moduli together with the Grüneisen parameter $(\gamma)$ at $0 \mathrm{GPa}$ and $0 \mathrm{~K}$. The $\Theta_{D}$ calculated from GIBBS code is shown in brackets.

\begin{tabular}{lllllll}
\hline & $\gamma$ & $\rho$ & $v_{t}$ & $v_{l}$ & $v_{m}$ & $\Theta_{D}$ \\
\hline LDA & 2.008 & 8.289 & 1621.8 & 2901.3 & 1805.4 & $162.6(160.9)$ \\
PBE & 1.993 & 7.606 & 1508.1 & 2722.2 & 1680.0 & $147.0(143.6)$ \\
PBESOL & 2.075 & 8.034 & 1565.9 & 2808.5 & 1743.6 & $155.4(153.0)$ \\
Exp. $^{\text {a }}$ & - & 7.805 & - & - & - & 75.9 \\
Exp. $^{\mathrm{b}}$ & - & 7.792 & - & - & - & - \\
\hline
\end{tabular}

${ }^{\mathrm{a}}$ Ref. [5]; ${ }^{\mathrm{b}}$ Ref. [7]. 

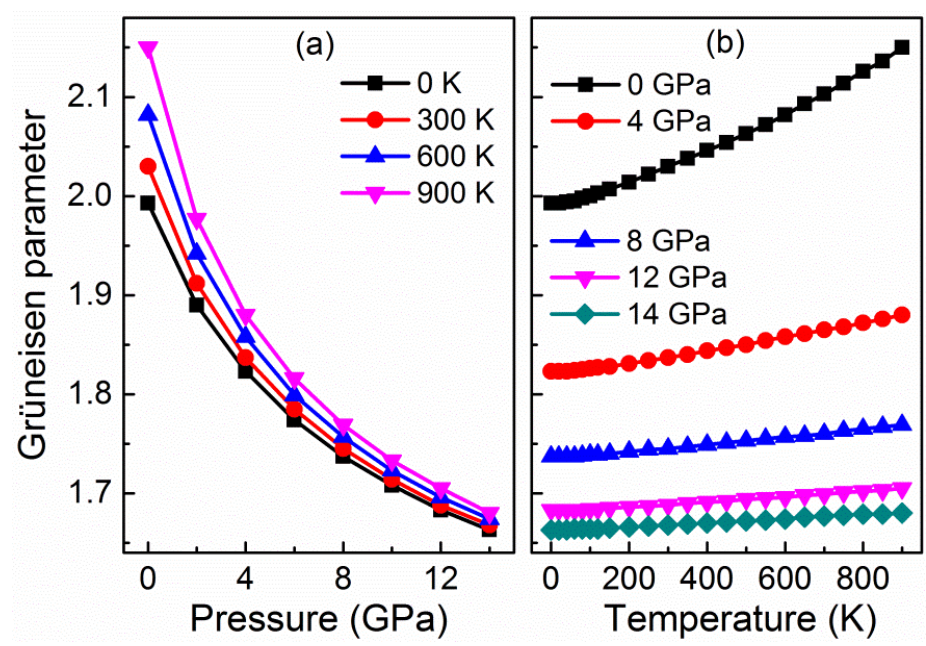

Fig. 14. (a) Pressure dependence and (b) temperature dependence of the Grüneisen parameter $(\gamma)$ for $\mathrm{Ba}_{2} \mathrm{Bi}_{3}$ within $\mathrm{PBE}$ calculations.

For the Debye temperature $\Theta_{D}$, another method on the basis of elastic moduli is usually used. As the vibrational excitations arise solely from acoustic vibrations at low temperatures, hence, the Debye temperature calculated from elastic moduli is the same as that determined from specific heat measurements at low temperatures [29]. The related equations are as follows $[42,43]$

$$
\begin{gathered}
v_{m}=\left[\frac{1}{3}\left(\frac{2}{v_{t}^{3}}+\frac{1}{v_{l}^{3}}\right)\right]^{-1 / 3}, \quad v_{t}=\sqrt{\frac{G}{\rho}} \text { and } v_{l}=\sqrt{\left(B+\frac{4}{3} G\right) / \rho}, \\
\Theta_{D}=\frac{h}{k}\left[\frac{3 n}{4 \pi}\left(\frac{N_{A} \rho}{M}\right)\right]^{1 / 3} v_{m} .
\end{gathered}
$$

Here, $v_{t}$ and $v_{l}$ are the transverse and longitudinal elastic wave velocity obtained from the shear modulus $G$ and bulk modulus $B$ of polycrystalline material, and $v_{m}$ is average elastic wave velocity. $\rho$ is the density, $h$ is Planck's constant, $k$ is Boltzmann's constant, $N_{A}$ is Avogadro's number, $M$ is the molecular weight and $n$ is the number of atoms in a unit cell.

The transverse, longitudinal, average elastic wave velocity $\left(v_{t}, v_{l}\right.$, and $\left.v_{m}\right)$, and Debye temperature $\left(\Theta_{D}\right)$ at $0 \mathrm{GPa}$ and $0 \mathrm{~K}$ are listed in Table 6. It can be seen from Table 6 that the results of $\Theta_{D}$ both from GIBBS code and elastic moduli are close to each other, implying the rationality of the $\Theta_{D}$ related calculations. Compared to the value of $\Theta_{D}$ in Ref. [5], the value from theoretical calculations is a little larger. Since 
the density of the $\mathrm{Ba}_{2} \mathrm{Bi}_{3}$ calculated from LDA, PBE and PBESOL is in good accordance with that in Ref. [5, 7], the larger $\Theta_{D}$ from theoretical calculations should be attributed to the theoretical elastic moduli. As is known, the elastic moduli are derived from elastic constants that stem from the structural parameters. Thus, the calculation accuracy of structural parameters has a great influence on the calculation of Debye temperature. Although the results of $\Theta_{D}$ from theoretical calculations are not comparable with that from experiment, the theoretical value is still meaningful to the analysis of the pressure effect on the superconductivity of the $\mathrm{Ba}_{2} \mathrm{Bi}_{3}$ (discussed below).

The pressure dependence of Debye temperature is shown in Fig. 11. It can be seen that the Debye temperature obtained from the two methods increases monotonously. The difference between the $\Theta_{D}$ obtained from the two theoretical methods is getting larger with the pressure. It's noted that the assumption of isotropic solid is adopted in the calculation of $\Theta_{D}$ in the GIBBS code. According to the analysis of anisotropy, the $\mathrm{Ba}_{2} \mathrm{Bi}_{3}$ is nearly isotropic at $0 \mathrm{GPa}$ and the pressure enhances the anisotropy, which can illuminate the similar results of $\Theta_{D}$ at $0 \mathrm{GPa}$ as well as the increasing difference with the pressure. Hence, for $\mathrm{Ba}_{2} \mathrm{Bi}_{3}$, the pressure dependence of $\Theta_{D}$ obtained from elastic moduli is more acceptable. The correlation between the pressure and the $\Theta_{D}$ obtained from elastic moduli satisfies the following relation

$$
\Theta_{D}(P)=147.775+5.29881 P-0.15952 P^{2} .
$$

Here, the unit of Debye temperature $\left(\Theta_{D}\right)$ is $\mathrm{K}$, and the unit of pressure $(P)$ is GPa.

\subsection{Superconductivity}

On the basis of the pressure dependence of total $N\left(E_{F}\right)$ and Debye temperature (as shown in Fig. 11), one can infer the pressure effect on the superconducting transition temperature $\left(T_{c}\right)$ of the $\mathrm{Ba}_{2} \mathrm{Bi}_{3}$. In the BCS strong coupling limit, $T_{c}$ can be estimated from McMillan formula [44]

$$
T_{c}=\frac{\Theta_{D}}{1.45} \exp \left[\frac{-1.04(1+\lambda)}{\lambda-\mu^{*}(1+0.62 \lambda)}\right],
$$


where $\lambda$ is the electron-phonon coupling constant given by $\lambda=N\left(E_{F}\right)\left\langle I^{2}\right\rangle /\left\langle M \omega^{2}\right\rangle$, $\left\langle I^{2}\right\rangle$ is the average over the Fermi surface of square of the electron-phonon matrix element, $M$ is the ionic mass, $\left\langle\omega^{2}\right\rangle$ is the square averaged phonon frequency which can be approximated as $\left\langle\omega^{2}\right\rangle=0.5 \Theta_{D}{ }^{2}, \mu^{*}$ is Coulomb pseudo-potential with the value usually used between 0.1 and 0.15 . From Ref. [44], one can obtain the empirical electron-phonon coupling constant $\lambda$ from the Debye temperature $\Theta_{D}$ and experimentally determined transition temperature $T_{c}$

$$
\lambda=\frac{1.04+\mu^{*} \ln \left(\Theta_{D} / 1.45 T_{c}\right)}{\left(1-0.62 \mu^{*}\right) \ln \left(\Theta_{D} / 1.45 T_{c}\right)-1.04} .
$$

If we take $T_{c}=4.4 \mathrm{~K}$ [5], $\Theta_{D}=147 \mathrm{~K}$ calculated on PBE and $\mu^{*}=0.15$, from Eq. (15), we can obtain $\lambda=0.84$ that is consistent with the result in Ref. [5], implying the rationality of the $\Theta_{D}$ related calculations.

It is obvious that in Eq. (14), $T_{c}$ is strongly dependent on $\Theta_{D}$ and $\lambda$. However, it is not easy to obtain $\lambda$ under various pressures due to the complicated electron-phonon matrix elements. The electron-phonon interactions are usually calculated from the response of the self-consistent potential to a lattice distortion [11] in combination with the density-functional perturbation theory (DFPT) [45]. That is a formidable task beyond the scope of this work. Therefore, we only discuss the probable tendency of the $T_{c}$ with the pressure. The analyzing methodology in Ref. [46] is employed to investigate how the variation of $\Theta_{D}$ affects the pressure dependence of $T_{c}$ of the $\mathrm{Ba}_{2} \mathrm{Bi}_{3}$. This methodology has been successfully applied to the discussion of the pressure effect on $T_{c}$ of the electron-phonon superconductor PdTe [39, 47, 48], likewise adopted in Ref. [49]. It's assumed that the $N\left(E_{F}\right),\left\langle I^{2}\right\rangle$ and $\mu^{*}$ are constant, with $\mu^{*}=0.15$. According to Eq. (14), the $\Theta_{D}$ at $14 \mathrm{GPa}$ gives a positive factor making $T_{c}$ grow to be about 1.3 times of $T_{c}$ at $0 \mathrm{GPa}$ in the linear term $\Theta_{D} / 1.45$. On the other hand, in the exponential term, the enhancement of $\Theta_{D}$ decreases $\lambda$ at $14 \mathrm{GPa}$ to about 0.6 times of $\lambda$ at $0 \mathrm{GPa}$. If we assume that the $\lambda$ at $0 \mathrm{GPa}$ is 0.84 , then the $T_{c}$ at 14 $\mathrm{GPa}$ is 0.18 times of $T_{c}$ at $0 \mathrm{GPa}$. So it's obvious that the $\Theta_{D}$ in the exponential term 
has a larger effect on the $T_{c}$ than that in the linear term. Synthetically, the enhancement of Debye temperature plays a negative role in the pressure dependence of $T_{c}$ for $\mathrm{Ba}_{2} \mathrm{Bi}_{3}$. Moreover, in this work, the reduction of $N\left(E_{F}\right)$ actually decreases the $\lambda$, which will obviously decrease the $T_{c}$. So if the $\left\langle I^{2}\right\rangle$ is less pressure sensitive, it is conservatively concluded that the $T_{c}$ of the $\mathrm{Ba}_{2} \mathrm{Bi}_{3}$ will decrease with the pressure.

\section{Conclusions}

The structural, mechanical, electronic and thermodynamic properties of the orthorhombic $\mathrm{Ba}_{2} \mathrm{Bi}_{3}$ superconductor have been systematically investigated under different pressures up to $14 \mathrm{GPa}$ by employing the first-principle method within the Local Density Approximation (LDA) and Generalized Gradient Approximation (GGA) approaches in combination with the quasi-harmonic Debye model. According to the mechanical stability criteria under pressure, the $\mathrm{Ba}_{2} \mathrm{Bi}_{3}$ can't be mechanically stable beyond the pressure of $14 \mathrm{GPa}$ any more. The ductility factors show that the $\mathrm{Ba}_{2} \mathrm{Bi}_{3}$ lies in between the brittle and ductile border line at $0 \mathrm{GPa}$ and the pressure can enhance the ductility. The elastic anisotropic factors indicate that the $\mathrm{Ba}_{2} \mathrm{Bi}_{3}$ is nearly isotropic at $0 \mathrm{GPa}$, and the pressure can make it more anisotropic. The electronic properties of the $\mathrm{Ba}_{2} \mathrm{Bi}_{3}$ are greatly influenced by $\mathrm{Ba}-d$ and $\mathrm{Bi}-p$ states and the pressure can make the delocalization behavior of electrons stronger. The pressure and temperature have opposite effect on the thermodynamic properties of the $\mathrm{Ba}_{2} \mathrm{Bi}_{3}$. According to the analysis of pressure effect on the total density of states at Fermi level and Debye temperature, it's conservatively concluded that the pressure can make the superconducting transition temperature of the $\mathrm{Ba}_{2} \mathrm{Bi}_{3}$ decrease.

Hopefully, the phonon related properties of the orthorhombic $\mathrm{Ba}_{2} \mathrm{Bi}_{3}$ should help to reveal the precise mechanism of the superconductivity under pressure, and may yield new and interesting information for exploring the physical properties of conventional and new high-temperature cuprates.

\section{Acknowledgements}

This work was financially supported by the Funds of the National Science 
Foundation of China (No. 11372096 and 11672100) and Program for Research Fund for the Doctoral Program of Higher Education of China. The authors gratefully acknowledge these financial supports.

\section{References}

[1] T. Kinjo, S. Kajino, T. Nishio, K. Kawashima, Y. Yanagi, I. Hase, T. Yanagisawa, S. Ishida, H. Kito, N. Takeshita, Superconductivity in LaBi3 with AuCu3-type structure, Superconductor Science and Technology, 29 (2016) 03LT02.

[2] S. Kushwaha, J. Krizan, J. Xiong, T. Klimczuk, Q. Gibson, T. Liang, N. Ong, R. Cava, Superconducting properties and electronic structure of NaBi, Journal of Physics: Condensed Matter, 26 (2014) 212201.

[3] S. Tencé, O. Janson, C. Krellner, H. Rosner, U. Schwarz, Y. Grin, F. Steglich, CoBi3-the first binary compound of cobalt with bismuth: high-pressure synthesis and superconductivity, Journal of Physics: Condensed Matter, 26 (2014) 395701.

[4] N. Haldolaarachchige, S. Kushwaha, Q. Gibson, R. Cava, Superconducting properties of BaBi3, Superconductor Science and Technology, 27 (2014) 105001.

[5] A. Iyo, Y. Yanagi, S. Ishida, K. Oka, Y. Yoshida, K. Kihou, C. Lee, H. Kito, N. Takeshita, I. Hase, Superconductivity at 4.4 K in Ba2Bi3, Superconductor Science and Technology, 27 (2014) 072001.

[6] S.C. Wimbush, Layers of intrigue: superconductivity at $4.4 \mathrm{~K}$ in Ba2Bi3, Superconductor Science and Technology, 27 (2014) 070501.

[7] S. Ponou, T.F. Fässler, Nonclassical bonding in the novel structure of Ba2Bi3 and unexpected site preference in the coloring variant Ba2BiSb2, Inorganic chemistry, 43 (2004) 6124-6126.

[8] Y. Mizuguchi, F. Tomioka, S. Tsuda, T. Yamaguchi, Y. Takano, Superconductivity at 27 K in tetragonal FeSe under high pressure, Applied Physics Letters, 93 (2008).

[9] H. Takahashi, K. Igawa, K. Arii, Y. Kamihara, M. Hirano, H. Hosono, Superconductivity at 43 K in an iron-based layered compound LaO1-xFxFeAs, Nature, 453 (2008) 376-378.

[10] T. Yajima, F. Takeiri, Y. Nozaki, Z. Li, T. Tohyama, M.A. Green, Y. Kobayashi, H. Kageyama, Superconductivity in the Hypervalent Compound Ba2Bi (Sb1-x Bi x) 2 with a Square-Honeycomb Lattice, Journal of the Physical Society of Japan, 83 (2014) 073705.

[11] P. Giannozzi, S. Baroni, N. Bonini, M. Calandra, R. Car, C. Cavazzoni, D. Ceresoli, G.L. Chiarotti, M. Cococcioni, I. Dabo, QUANTUM ESPRESSO: a modular and open-source software project for quantum simulations of materials, Journal of Physics: Condensed Matter, 21 (2009) 395502.

[12] D.M. Ceperley, B. Alder, Ground state of the electron gas by a stochastic method, Physical Review Letters, 45 (1980) 566.

[13] J.P. Perdew, A. Zunger, Self-interaction correction to density-functional approximations for many-electron systems, Physical Review B, 23 (1981) 5048.

[14] J.P. Perdew, K. Burke, M. Ernzerhof, Generalized gradient approximation made simple, Physical review letters, 77 (1996) 3865.

[15] J.P. Perdew, A. Ruzsinszky, G.I. Csonka, O.A. Vydrov, G.E. Scuseria, L.A. Constantin, X. Zhou, K. Burke, Restoring the density-gradient expansion for exchange in solids and surfaces, Physical Review Letters, 100 (2008) 136406.

[16] K.F. Garrity, J.W. Bennett, K.M. Rabe, D. Vanderbilt, Pseudopotentials for high-throughput DFT 
calculations, Computational Materials Science, 81 (2014) 446-452.

[17] K. Lejaeghere, G. Bihlmayer, T. Björkman, P. Blaha, S. Blügel, V. Blum, D. Caliste, I.E. Castelli, S.J. Clark, A. Dal Corso, Reproducibility in density functional theory calculations of solids, Science, 351 (2016) aad3000.

[18] H.J. Monkhorst, J.D. Pack, Special points for Brillouin-zone integrations, Physical Review B, 13 (1976) 5188-5192.

[19] Dewhurst J K et al. ELK FP-LAPW code, version 3.3.17 (2016); http://elk.sourceforge.net. Date of access: 2016.03.20.

[20] F. Birch, Finite strain isotherm and velocities for single- crystal and polycrystalline $\mathrm{NaCl}$ at high pressures and $300^{\circ} \mathrm{K}$, Journal of Geophysical Research: Solid Earth (1978-2012), 83 (1978) 1257-1268.

[21] B. Karki, L. Stixrude, S. Clark, M. Warren, G. Ackland, J. Crain, Elastic properties of orthorhombic $\mathrm{MgSiO} 3$ perovskite at lower mantle pressures, American Mineralogist, 82 (1997) 635-638.

[22] B. Karki, L. Stixrude, S. Clark, M. Warren, G. Ackland, J. Crain, Structure and elasticity of MgO at high pressure, American Mineralogist, 82 (1997) 51-60.

[23] R.M. Wentzcovitch, N.L. Ross, G. Price, Ab initio study of $\mathrm{MgSiO} 3$ and $\mathrm{CaSiO} 3$ perovskites at lower-mantle pressures, Physics of the Earth and Planetary Interiors, 90 (1995) 101-112.

[24] J.F. Nye, Physical properties of crystals: their representation by tensors and matrices, Oxford university press, 1985.

[25] G. Sin'Ko, N. Smirnov, Ab initio calculations of elastic constants and thermodynamic properties of bcc, fcc, and hcp Al crystals under pressure, Journal of Physics: Condensed Matter, 14 (2002) 6989.

[26] G. Sin'ko, N. Smirnov, Relative stability and elastic properties of hcp, bcc, and fcc beryllium under pressure, Physical Review B, 71 (2005) 214108.

[27] Z.-j. Wu, E.-j. Zhao, H.-p. Xiang, X.-f. Hao, X.-j. Liu, J. Meng, Crystal structures and elastic properties of superhard Ir N 2 and Ir N 3 from first principles, Physical Review B, 76 (2007) 054115.

[28] R. Hill, The elastic behaviour of a crystalline aggregate, Proceedings of the Physical Society. Section A, 65 (1952) 349.

[29] P. Ravindran, L. Fast, P.A. Korzhavyi, B. Johansson, J. Wills, O. Eriksson, Density functional theory for calculation of elastic properties of orthorhombic crystals: application to TiSi2, Journal of Applied Physics, 84 (1998) 4891-4904.

[30] R. Golesorkhtabar, P. Pavone, J. Spitaler, P. Puschnig, C. Draxl, ElaStic: A tool for calculating second-order elastic constants from first principles, Computer Physics Communications, 184 (2013) 1861-1873.

[31] S. Margadonna, Y. Takabayashi, Y. Ohishi, Y. Mizuguchi, Y. Takano, T. Kagayama, T. Nakagawa, M. Takata, K. Prassides, Pressure evolution of the low-temperature crystal structure and bonding of the superconductor FeSe (T c= 37 K), Physical Review B, 80 (2009) 064506.

[32] I. Frantsevich, F. Voronov, S. Bokuta, I. Frantsevich, Elastic constants and elastic moduli of metals and insulators handbook, Naukova Dumka, Kiev, (1983) 60-180.

[33] S. Pugh, XCII. Relations between the elastic moduli and the plastic properties of polycrystalline pure metals, Philosophical Magazine, 45 (1954) 823-843.

[34] X. Zhang, J. Qin, J. Ning, X. Sun, X. Li, M. Ma, R. Liu, First principle study of elastic and thermodynamic properties of FeB4 under high pressure, Journal of Applied Physics, 114 (2013) 183517. 
[35] D. Chung, W. Buessem, F. Vahldiek, S. Mersol, Anisotropy in single crystal refractory compounds, Plenum Press, New York, 1968 p. 217, (1968).

[36] S.I. Ranganathan, M. Ostoja-Starzewski, Universal elastic anisotropy index, Physical Review Letters, 101 (2008) 055504.

[37] Y. Ding, B. Xiao, Anisotropic elasticity, sound velocity and thermal conductivity of TiO 2 polymorphs from first principles calculations, Computational Materials Science, 82 (2014) 202-218.

[38] M. Blanco, E. Francisco, V. Luana, GIBBS: isothermal-isobaric thermodynamics of solids from energy curves using a quasi-harmonic Debye model, Computer Physics Communications, 158 (2004) 57-72.

[39] J.-J. Cao, X.-F. Gou, X.-L. Yuan, The elastic, electronic and thermodynamic properties of PdTe under high pressure from first-principles calculations, Physica C: Superconductivity and its Applications, 509 (2015) 34-41.

[40] R. Yang, C. Zhu, Q. Wei, Z. Du, Investigations on structural, elastic, thermodynamic and electronic properties of TiN, Ti $2 \mathrm{~N}$ and Ti $3 \mathrm{~N} 2$ under high pressure by first-principles, Journal of Physics and Chemistry of Solids, 98 (2016) 10-19.

[41] C. Kittel, P. McEuen, Introduction to solid state physics, Wiley New York, 1976.

[42] O.L. Anderson, A simplified method for calculating the Debye temperature from elastic constants, Journal of Physics and Chemistry of Solids, 24 (1963) 909-917.

[43] E. Screiber, O. Anderson, N. Soga, Elastic constants and their measurements, New York: McGrawHill, (1973).

[44] W. McMillan, Transition temperature of strong-coupled superconductors, Physical Review, 167 (1968) 331.

[45] S. Baroni, S. de Gironcoli, A. Dal Corso, P. Giannozzi, Phonons and related crystal properties from density-functional perturbation theory, Reviews of Modern Physics, 73 (2001) 515.

[46] W. Zhang, X.-R. Chen, L.-C. Cai, F.-Q. Jing, Elastic and electronic properties of perovskite type superconductor MgCNi3 under pressure, Journal of Physics: Condensed Matter, 20 (2008) 325228.

[47] J.-J. Cao, X.-F. Gou, First-principles study of electronic structure, phonons and electron-phonon interaction in hexagonal PdTe, Physica C: Superconductivity and its Applications, 520 (2016) 19-23.

[48] J. Chen, X. Wang, Superconductivity origin of PdTe and pressure effect: Insights from first-principles investigation, Solid State Sciences, 52 (2016) 23-28.

[49] M. Ali, A. Islam, M. Ali, Ni-rich Nitrides ANNi 3 ( $\mathrm{A}=\mathrm{Pt}, \mathrm{Ag}, \mathrm{Pd})$ in Comparison with Superconducting ZnNNi 3, Journal of Scientific Research, 4 (2011) 1. 